\title{
Influence of external electric fields on multi-photon transitions between the 2s, 2p and 1s levels for hydrogen and antihydrogen atoms and hydrogen-like ions.
}

\author{
D. Solovyev ${ }^{1}$, L. Labzowsky ${ }^{1,2}$, G. Plunien ${ }^{3}$ and V. Sharipov ${ }^{1}$ \\ ${ }^{1}$ V. A. Fock Institute of Physics, St. Petersburg State University, \\ Petrodvorets, Ulianovskaya 1, 198504, St. Petersburg, Russia \\ ${ }^{2}$ Petersburg Nuclear Physics Institute, 188300, Gatchina, St. Petersburg, Russia \\ ${ }^{3}$ Technische Universität Dresden, Mommsenstrasse 13, D-01062, Dresden, Germany.
}

\begin{abstract}
One- and two-photon transitions in the hydrogen atom are analytically evaluated in the absence and in the presence of an external electric field. The emission probabilities are different for the hydrogen $(\mathrm{H})$ and antihydrogen $(\overline{\mathrm{H}})$ atoms due to the existence of contributions, linear in electric field. The magnitude of these contributions is evaluated within the nonrelativistic limit. The Coulomb Green function method is applied. Different nonrelativistic "forms" for the decay probabilities in combination with different gauge choices are considered. The three-photon E1E1E1 2p-1s transition probability is also evaluated and possible applications of the results are discussed.

PACS number(s): 31.30. Jv, 12.20. Ds, 31.15.-p
\end{abstract}

\section{INTRODUCTION}

Recent experimental success in the production of anti-hydrogen atoms [1, 2] opens realistic chances for a comparison of the atomic spectra for hydrogen $(\mathrm{H})$ and anti-hydrogen $(\overline{\mathrm{H}})$ atoms. One of the purposes for this comparison is the search for the CPT-violating effects [3]. The possibility of the CPT-tests is connected with the modern extra-accurate resonance frequency measurements in hydrogen [4, 5]. In [6]-[8] it was shown that a specific difference in the $\mathrm{H}$ and $\overline{\mathrm{H}}$ atomic spectra arises even in the absence of the CPT-violation, if an external electric field is present. In principle, a difference arises also for the frequency measurements if nonresonant (NR) corrections are taken into account. Actually, the NR corrections define the natural limit up to which the frequency measurements have sense: beyond this limit the spectral line profile cannot be defined by the two parameters: resonance frequency and width. NR corrections are not just corrections to the level energy, but rather corrections to the spectral line profile. These corrections for the atoms placed in an external electric field contain terms, linear in the electric field. Therefore NR corrections appear to be different for $\mathrm{H}$ and $\overline{\mathrm{H}}$ atoms. However, this difference is very small and hence the existence of the electric stray field should not become a serious problem in performing experiments searching for CPT-violating effects. Another difference in spectroscopic properties of $\mathrm{H}$ and $\overline{\mathrm{H}}$ atoms in an external electric field is the difference in the transition probabilities, also caused by the terms, linear in electric field. This effect is much larger than the difference in the NR corrections and was not yet discussed in detail.

In the present paper we will investigate specially the difference in the one-photon $2 \mathrm{~s}-1 \mathrm{~s}$ emission probabilities in $\mathrm{H}$ and $\overline{\mathrm{H}}$ atoms in an external electric field and will find the optimal conditions for observing this effect. This investigation should confirm our understanding of fundamental symmetries in physics. Thus, going over from $\mathrm{H}$ to $\overline{\mathrm{H}}$ and changing simultaneously the sign of the electric field should not change the atomic spectra provided that CPT symmetry is conserved.

The one-photon decay of the $2 \mathrm{~s}$ state of the $\mathrm{H}$ atom in an external electric field was utilized in the experiments [4, 5] for the registration of the $2 \mathrm{~s}-1 \mathrm{~s}$ two-photon resonance absorption. The difference of the one-photon $2 \mathrm{~s}-1 \mathrm{~s}$ decay rate for $\mathrm{H}$ and $\overline{\mathrm{H}}$ atoms in an external electric field can be observable in the similar experiments with $\overline{\mathrm{H}}$ atoms.

The probabilities for the spontaneous two-photon decay in hydrogen atoms and hydrogen-like ions are under investigation since the theoretical formalism has been introduced by Göppert-Mayer [9] and the first evaluation for the two-photon E1E1 transition $2 \mathrm{~s} \rightarrow 2 \gamma(\mathrm{E} 1)+1 \mathrm{~s}$ has been presented by Breit and Teller [10]. A highly accurate calculation of the E1E1 - transition probability has been performed by Klarsfeld [11]. Recently Jentschura [12] performed a complete evaluation of the radiative corrections and presented more accurate value of the E1E1 two-photon decay probability. The double- and triple-photon decays of metastable ${ }^{3} P_{0}$ atomic state were considered in [13]. The present paper is devoted also to evaluation of the probabilities for two-photon decays $2 \mathrm{p} \rightarrow \gamma(\mathrm{E} 1)+\gamma(\mathrm{M} 1)+1 \mathrm{~s}$ and $2 \mathrm{p} \rightarrow \gamma(\mathrm{E} 1)+\gamma(\mathrm{E} 2)+1 \mathrm{~s}$. Evaluations of these two transitions have been first accomplished in [14, 15] for hydrogen-like systems with nuclear charge numbers $Z$ within the range $1 \leq Z \leq 100$ by pure numerical methods. Here we present analytic calculations in the nonrelativistic limit and compare them with corresponding numerical results. For performing the summations over intermediate states (i.e. over the complete set of solutions of the Schrödinger equation describing electrons in the Coulomb field of the nucleus) we employ the Coulomb Green function [16]. The Green function method has been first applied for deriving the general expression for the two-photon decay probability in $\mathrm{H}$ atom and $\mathrm{H}$-like ions in [17, 18]. An alternative approach applicable for arbitrary states based on Schwinger's analytical representation of the Coulomb Green function has been presented in [19, 20].

As it has been indicated in [21], the nonrelativistic behavior of E1M1 transitions as a function of $Z$ with the neglect of the 
interelectron interaction should be $W^{\mathrm{E} 1 \mathrm{M} 1}=(8 / 9 \pi)(\alpha Z)^{12} / 100$ for the helium-like systems. This very small value arises due to the cancellation of contributions of the leading terms $2 \mathrm{p}_{1 / 2}$ and $2 \mathrm{p}_{3 / 2}$ in the summation over intermediate np-states. However, we should note that this result yields only a minor contribution for small nuclear charges $Z$ when it is evaluated within the "velocity" gauge [21]. In this case a major contribution arises from the negative-energy intermediate states and scales like $(\alpha Z)^{8}$ in atomic units [14], [15].

The two-photon $2 \mathrm{p}_{1 / 2} \rightarrow \gamma(\mathrm{E} 1)+\gamma(\mathrm{E} 2)+1$ s transition rate for hydrogen and hydrogen-like ions is proportional to $(\alpha Z)^{8}$ in atomic units. Thus the two-photon transitions represent higher-order corrections to the life time of the $2 \mathrm{p}_{1 / 2}$-level when compared to the lowest order $(\alpha Z)^{3} \ln (\alpha Z)$ (in relativistic unit) radiative corrections derived in references [22], [23]. A direct observation of the influence of the two photon $2 \mathrm{p}_{1 / 2} \rightarrow \gamma(\mathrm{E} 1)+\gamma(\mathrm{E} 2)+1 \mathrm{~s}$ transitions in the $\mathrm{H}$ atom does not look feasible due to the huge background arising from the one-photon transition $2 \mathrm{p}_{1 / 2} \rightarrow \gamma(\mathrm{E} 1)+1$ s. However, two-photon decays of the $2 \mathrm{p}_{1 / 2}$-level could be observed in coincidence experiments.

In this paper we reevaluate the two-photon decay rates of the 2p-state in hydrogen, deriving the E1E2 and E1M1 contributions to the two-photon emission processes by means of analytical methods. These calculations are performed within different gauges and employing different forms for the expression of the transition probability (see [24], [25]).

We also perform the calculation of two-photon transition probabilities from the $2 \mathrm{~s}$ and $2 \mathrm{p}_{1 / 2}$ hydrogenic levels in the presence of an external electric field. The mixing of the $2 \mathrm{~s}$ and $2 \mathrm{p}_{1 / 2}$ levels results in additional terms, linear in electric field. The evaluation is performed within the nonrelativistic limit by the Coulomb Green function method [18] in the "length" gauge. These terms linear in the electric field lead to the difference of the corresponding probabilities, see [26]-[28], for the hydrogen and anti-hydrogen atoms. The magnitude of these contributions is evaluated and the possibility of the observation of this effect is discussed. It is important to stress, that these linear field corrections even in a very small stray electric field can be larger than the radiative correction to the decay of $2 \mathrm{~s}$ level, evaluated in [12].

Finally, we evaluate a three-photon emission probability for the hydrogen atom. This question was never debated in literature.

The recent success in observation of the cosmic microwave background temperature and polarization anisotropy draw attention to the details of the cosmological hydrogen recombination history. This, in turn, required the accurate knowledge of the twophoton decay processes in hydrogen (see [29, 30] for details and references). The main interest concentrates around the 2s-1s E1E1 two-photon transition and the two-photon decays for the ns, nd excited states.

The smallness of the numerical coefficients in E1E2 and E1M1 two-photon expressions excludes any significant role of these transitions for astrophysics. The same can be stated for the 3-photon 3E1 2 $\mathrm{p}_{1 / 2}-1 \mathrm{~s}$ decay. However the values for the $2 \mathrm{p}_{1 / 2}-1 \mathrm{~s}$ E1M1 and E1E2 transition rates appeared to be important for the determination of the theoretical accuracy limits [31, 32] for frequency measurements by means of optical resonance experiments with hydrogen [4, 5].

The following notations will be used throughout this paper. The $2 \mathrm{p}_{1 / 2}$ level is labelled as $2 \mathrm{p}$ because the $2 \mathrm{p}_{3 / 2}$ level will not be considered. Vectors with 3 components are in bold. Angular part of any vector $\mathbf{r}$ is defined by the unit vector $\mathbf{n}_{\mathbf{r}}$ directed the same as $\mathbf{r}$. The Clebsh-Gordon coefficients $C_{\ell_{1} m_{1} \ell_{2} m_{2}}^{j m}$ are introduced according to [33]. Other notations are the following:

$$
\begin{aligned}
& \Delta=\sqrt{\Delta E_{\mathrm{L}}^{2}+\frac{1}{4} \Gamma_{2 \mathrm{p}}^{2}}, \\
& w^{(1 \gamma)}=\sqrt{W_{2 \mathrm{~s}}^{(1 \gamma)} / W_{2 \mathrm{p}}^{(1 \gamma)}}, \\
& w^{(2 \gamma)}=\sqrt{W_{2 \mathrm{~s}}^{(2 \gamma)} / W_{2 \mathrm{p}}^{(2 \gamma)}} .
\end{aligned}
$$

Here $\Delta E_{\mathrm{L}}$ is the Lamb shift, $\Gamma_{2 \mathrm{p}}$ is the width of the $2 \mathrm{p}$ state, $W_{2 \mathrm{~s}}^{(1 \gamma),(2 \gamma)}$ and $W_{2 \mathrm{p}}^{(1 \gamma),(2 \gamma)}$ are the one-photon and two-photon decay probabilities for the corresponding $2 \mathrm{~s}$ and $2 \mathrm{p}$ levels. The value $W_{2 \mathrm{p}}^{(2 \gamma)}=W_{2 \mathrm{p}}^{(\mathrm{E} 1 \mathrm{E} 2)}+W_{2 \mathrm{p}}^{(\mathrm{E} 1 \mathrm{M} 1)}$ includes the probabilities for $2 \mathrm{p} \rightarrow \gamma(\mathrm{E} 1)+\gamma(\mathrm{E} 2)+1 \mathrm{~s}$ and $2 \mathrm{p} \rightarrow \gamma(\mathrm{E} 1)+\gamma(\mathrm{M} 1)+1$ s decays.

\section{S DECAY RATE FOR HYDROGEN AND ANTI-HYDROGEN ATOMS IN EXTERNAL ELECTRIC FIELDS}

In the absence of an electric field, the M1 transition $2 \mathrm{~s} \rightarrow 1 \mathrm{~s}+\gamma$ - strongly forbidden in the nonrelativistic limit - was first evaluated by Breit and Teller [10]. The value, obtained in [10] was later improved by Drake [34] (see also [35]). In [34, 35] relativistic corrections to the Schrödinger wave functions and to the M1 photon emission operator were taken into account. Neglecting these corrections the $2 \mathrm{~s} \rightarrow 1 \mathrm{~s}+\gamma$ transition probability turns to be zero due to the orthogonality of the radial wave functions. Accurate fully relativistic calculations for the $2 \mathrm{~s} \rightarrow 1 \mathrm{~s}+\gamma$ transition for the hydrogen-like ions with arbitrary nuclear charge $\mathrm{Z}$ values were performed by Johnson [36]. 
Within QED theory the S-matrix element for the one-photon emission process reads [37]

$$
S_{A^{\prime} A}=\frac{e \sqrt{4 \pi}}{\sqrt{2 \omega}} \int d x \bar{\psi}_{A^{\prime}}(x) e_{\mu}^{*} \gamma^{\mu} e^{-i(\mathbf{k r}-\omega t)} \psi_{A}(x),
$$

where $\mathbf{k}$ is the wave vector of the photon, $\omega=|\mathbf{k}|$ is the photon frequency, $x=(\mathbf{r}, t)$ is the 4-vector of space-time coordinates, $e_{\mu}$ is the 4-vector of the photon polarization, $\psi_{A}(x)$ is the Dirac wave function for the bound electron in an atom, $\gamma_{\mu}$ are the Dirac matrices and $A^{\prime}, A$ correspond to the relevant quantum numbers characterizing the final and initial states of the electron in an atom. In Eq. (4) $e$ is the electron charge and the relativistic units $\hbar=c=1$ are used throughout this section.

We integrate over the time variable $t$ in Eq. (4) and employ the relation

$$
S_{A^{\prime} A}=-2 \pi i \delta\left(E_{A^{\prime}}-E_{A}-\omega\right) U_{A^{\prime} A}
$$

for the transition amplitude $U_{A^{\prime} A}$. In what follows, we define also the amplitude $U_{A^{\prime} A}(\mathbf{k}, \mathbf{e})$ as

$$
U_{A^{\prime} A}(\mathbf{k}, \mathbf{e})=\frac{\sqrt{2 \pi \omega}}{e} U_{A^{\prime} A}=\left\langle A^{\prime}\left|\mathbf{e} \alpha e^{-i \mathbf{k r}}\right| A\right\rangle .
$$

Here $\mathbf{e}$ is the transverse polarization vector and $\alpha$ are the Dirac matrices.

Transition probability integrated over the photon emission directions and summed over the polarizations is

$$
W_{A^{\prime} A}^{(1 \gamma)}=\frac{e^{2} \omega_{A A^{\prime}}}{2 \pi} \sum_{\mathbf{e}} \int d \mathbf{n}_{\mathbf{k}}\left|U_{A^{\prime} A}(\mathbf{k}, \mathbf{e})\right|^{2},
$$

$\omega_{A A^{\prime}}=E_{A}-E_{A^{\prime}}, E_{A^{\prime}}, E_{A}$ are the Dirac eigenvalues for the atomic electron, $\left\langle A^{\prime}|\ldots| A\right\rangle$ is the matrix element with the Dirac 4-component wave functions. Summation over e implies the transversality condition ek $=0$.

Within the Pauli approximation Eq. (7) reduces to [35]:

$$
W_{A^{\prime} A}^{(1 \gamma)}=\frac{e^{2} \omega_{A A^{\prime}}}{2 \pi} \sum_{\mathbf{e}} \int d \mathbf{n}_{\mathbf{k}}\left|U_{A^{\prime} A}^{\mathrm{P}}(\mathbf{k}, \mathbf{e})\right|^{2}
$$

with the transition amplitude in the Pauli approximation defined via the matrix element of the corresponding emission operator

$$
U_{A^{\prime} A}^{\mathrm{P}}(\mathbf{k}, \mathbf{e})=\left((\mathbf{e} \hat{\mathbf{p}}+i \mathbf{e}[\mathbf{k} \times \hat{\mathbf{s}}]) e^{-i \mathbf{k r}}\right)_{A^{\prime} A} .
$$

The relation Eq. (9) involves the nonrelativistic electron momentum operator $\hat{\mathbf{p}}=-i \nabla$, the electron spin operator $\hat{\mathbf{s}}=\frac{1}{2} \sigma(\sigma$ are the Pauli matrices) and $(\ldots)_{A^{\prime} A} \equiv\left(A^{\prime}|\ldots| A\right)$ denotes the matrix element with the Schrödinger wave functions. The first term of the integrand in Eq. (8) describes the E1 photon emission, that in case of $A^{\prime}=1 \mathrm{~s}, A=2 \mathrm{~s}$ is forbidden by parity; The second term corresponds to the M1 photon emission. Due to the orthogonality of the radial wave functions $\psi_{2 \mathrm{~s}}$ and $\psi_{1 \mathrm{~s}}$ the M1 transition probability as described by Eq. (8) is nonzero only due to the factor $e^{-i \mathbf{k r}}$ and due to the relativistic corrections to the Schrödinger wave functions. For the atomic electron $r \simeq a_{0}=1 / m \alpha Z\left(a_{0}\right.$ is the Bohr's radius, $m$ is the electron mass, $\alpha \approx 1 / 137$ is the fine structure constant), $\omega \approx m(\alpha Z)^{2}$ and $\mathbf{k r} \approx \alpha$. Thus, one can restrict the consideration to the lowest nonvanishing term of the multipole expansion of $e^{-i \mathbf{k r}}$, which turns to be of the same order of magnitude as the relativistic corrections to the wave functions.

The zero-order wave functions which should be employed for the evaluation of the matrix elements in Eq. (8) look like

$$
\psi_{A}(\mathbf{r}) \equiv \psi_{n j l m}(\mathbf{r})=\sum_{m_{l} m_{s}} C_{\ell m_{\ell} \frac{1}{2} m_{s}}^{j m} R_{n l}(r) Y_{l m_{l}}\left(\mathbf{n}_{\mathbf{r}}\right) \chi_{m_{s}},
$$

where the standard set of one-electron quantum numbers is introduced: Principal quantum number $n$, total electron angular momentum $j$ and its projection $m$, orbital electron angular momentum $l$ and its projection $m_{l}$, spin projection $m_{s}$. The function $\chi_{m_{s}}$ is the Pauli spinor. For the $2 \mathrm{~s}$ and $1 \mathrm{~s}$ electron states $l=0$ and the transition amplitude in Eq. (8) reduces to [35]

$$
\begin{array}{r}
U_{2 \mathrm{~s} m_{s} 1 \mathrm{~s} m_{s}^{\prime}}^{\mathrm{P}}=-i k^{2}\left\langle m_{s}|\mathbf{e}[\mathbf{k} \times \sigma]| m_{s}^{\prime}\right\rangle R_{2 \mathrm{~s} 1 \mathrm{~s}}, \\
R_{2 \mathrm{~s} 1 \mathrm{~s}}=\int_{0}^{\infty} R_{2 \mathrm{~s}}(r) R_{1 \mathrm{~s}}(r) r^{4} d r,
\end{array}
$$


where $\left|m_{s}\right\rangle$ are the spin wave functions, $R_{2 \mathrm{~s}}(r), R_{1 \mathrm{~s}}(r)$ are the nonrelativistic radial wave functions. Inserting Eq. (11) into Eq. (8), we have to sum over $m_{s}^{\prime}$ and average over $m_{s}$. For summation over e the standard formula

$$
\sum_{\mathbf{e}} e_{i} e_{k}=\delta_{i k}-n_{i} n_{k}
$$

is used, where $n_{i}$ and $n_{k}$ are the Cartesian components of the vector $\mathbf{n}_{\mathbf{k}}$. After integration over the photon emission directions $\mathbf{n}_{\mathbf{k}}$ one finds

$$
W_{2 \mathrm{~s} 1 \mathrm{~s}}^{(1 \gamma)}=\frac{3^{5}}{2^{21}} \alpha m^{5}(\alpha Z)^{14} R_{2 \mathrm{~s} 1 \mathrm{~s}}^{2}
$$

respectively. The evaluation of the radial integral in Eq. (14) results

$$
W_{2 \mathrm{~s} 1 \mathrm{~s}}^{(1 \gamma)}=\frac{1}{972} m \alpha(\alpha Z)^{10}=2.8 \cdot 10^{-6} \mathrm{~s}^{-1}
$$

The decay rate of the $2 \mathrm{~s}$ state of a hydrogen atom in the presence of an external electric field was studied in [26]-[28]. The external electric field mixes the states 2 s and 2p. A $100 \%$ mixing occurs in a field with the strength $D_{\mathrm{c}}=475 \mathrm{~V} / \mathrm{cm}[38]$. In what follows we will consider weaker fields $D<D_{\text {c }}$ such that admixtures of all other states, apart from $2 \mathrm{p}$ can be neglected. The ground state $1 \mathrm{~s}$ will be assumed unaffected by the field. We will denote the mixed states $2 \mathrm{~s}$ and $2 \mathrm{p}$ as $\overline{2 \mathrm{~s}}$ and $\overline{2 \mathrm{p}}$, respectively. For the wave function of the $\overline{2 s}$ state we can write [27]

$$
\left|\overline{2 \mathrm{~s}} m_{s}\right\rangle=\left|2 \mathrm{~s} m_{s}\right\rangle+\eta \sum_{m_{s}^{\prime \prime}}\left\langle 2 \mathrm{p} m_{s}^{\prime \prime}|e \mathbf{D r}| 2 \mathrm{~s} m_{s}\right\rangle\left|2 \mathrm{p} m_{s}^{\prime \prime}\right\rangle
$$

where $\eta=\left(\Delta E_{\mathrm{L}}+i \Gamma_{2 \mathrm{p}} / 2\right)^{-1}, \mathbf{D}$ is the electric field vector.

The transition amplitude Eq. (11) in an external electric field looks like

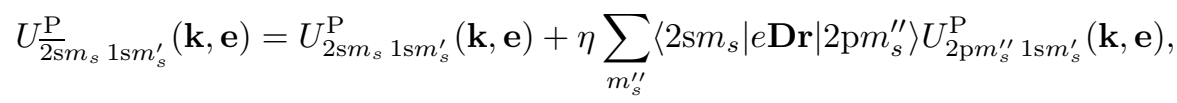

where

$$
U_{2 \mathrm{p} m_{s}^{\prime \prime} 1 \mathrm{~s} m_{s}^{\prime}}^{\mathrm{P}}(\mathbf{k}, \mathbf{e})=i m \omega_{2 \mathrm{~s} 1 \mathrm{~s}}\left\langle 2 \mathrm{p} m_{s}^{\prime \prime}|\mathbf{e r}| 1 \mathrm{~s} m_{s}^{\prime}\right\rangle
$$

Direct evaluation of the integral in Eq. (18) results in

$$
U_{2 \mathrm{p} m_{s}^{\prime \prime} 1 \mathrm{~s} m_{s}^{\prime}}^{\mathrm{P}}(\mathbf{k}, \mathbf{e})=3 i m \omega_{2 \mathrm{~s} 1 \mathrm{~s}} \sum_{q}(-1)^{q} e_{q} C_{1 \bar{q} \frac{1}{2} m_{s}^{\prime}}^{\frac{1}{2} m_{s}^{\prime \prime}},
$$

where $e_{q}$ are the spherical components of the vector e and $\bar{q}=-q$. Similarly,

$$
\left\langle 2 \mathrm{~s} m_{s}|e \mathbf{D r}| 2 \mathrm{p} m_{s}^{\prime \prime}\right\rangle=3 e \sum_{q^{\prime}}(-1)^{q^{\prime}} D_{q} C_{1 \bar{q}^{\prime} \frac{1}{2} m_{s}^{\prime}}^{\frac{1}{2} m^{\prime \prime}}
$$

Further evaluation requires the insertion of the amplitude Eq. (11) in Eq. (7) and summation over e. For this purpose the formula Eq. (13) should be used and then the representation of the scalar and vector products in spherical components should be employed. The final result is (here we do not integrate over the directions $\mathbf{n}_{\mathbf{k}}$ ):

$$
d W_{\overline{2 \mathrm{~s}} 1 \mathrm{~s}}^{(1 \gamma)}\left(\mathbf{n}_{\mathbf{k}}\right)=\frac{3}{8 \pi} W_{2 \mathrm{~s} 1 \mathrm{~s}}^{(1 \gamma)}\left[1+e \mathbf{D n}_{\mathbf{k}} \frac{\Gamma_{2 \mathrm{p}}}{w^{(1 \gamma)} \Delta^{2}}+\frac{e^{2} D^{2}}{\left(w^{(1 \gamma)} \Delta\right)^{2}}\right] d \mathbf{n}_{\mathbf{k}}
$$

Although formula Eq. (21) was obtained earlier in [26] and [27], [28]. We present here our way for its derivation.

The formal T-noninvariance of the factor $\mathbf{D} \mathbf{n}_{\mathbf{k}}$ in Eq. (21) ( $\mathbf{n}_{\mathbf{k}}$ and $\mathbf{D}$ are T-odd and T-even vectors, respectively) is compensated by the dependence on $\Gamma_{2 p}$; This is the imitation of T-noninvariance in unstable systems, as predicted by Zeldovich [39]. Rewriting Eq. [21] into the form [26]

$$
d W_{\overline{2 \mathrm{~s}} 1 \mathrm{~s}}^{(1 \gamma)}=W_{0}\left[1 \mp \beta(D) \mathbf{n}_{\mathbf{D}} \mathbf{n}_{\mathbf{k}}\right] d \mathbf{n}_{\mathbf{k}}
$$


where

$$
\begin{gathered}
W_{0}=\frac{3}{8 \pi} W_{2 \mathrm{~s} 1 \mathrm{~s}}^{(1 \gamma}\left(1+\frac{e^{2} D^{2}}{\left(w^{(1 \gamma)} \Delta\right)^{2}}\right), \\
\beta(D)=\frac{|e| D \Gamma_{2 \mathrm{p}} w^{(1 \gamma)}}{\left(w^{(1 \gamma)} \Delta\right)^{2}+e^{2} D^{2}},
\end{gathered}
$$

we find the maximum value $\beta(D)$ at [26]

$$
D_{\max }=\frac{w^{(1 \gamma)} \Delta}{|e|} \approx 0.3 \cdot 10^{-4} \mathrm{~V} / \mathrm{cm}
$$

The value $\beta_{\max }=\beta\left(D_{\max }\right)$ is equal to

$$
\beta_{\max }=\frac{\Gamma_{2 \mathrm{p}}}{2 \Delta} \approx \frac{1}{20}
$$

The $(-)$ and $(+)$ signs in Eq. (22) correspond to the $\mathrm{H}$ and $\overline{\mathrm{H}}$ atoms, respectively.

The relative difference for the decay rates in $\mathrm{H}$ and $\overline{\mathrm{H}}$ atoms at the maximum value $D_{\max }$ equals to:

$$
\frac{d W_{\overline{2 \mathrm{~s}} 1 \mathrm{~s}}^{(1 \gamma)}(\mathrm{H})}{d W_{2 \mathrm{~s} 1 \mathrm{~s}}^{(1 \gamma)}}-\frac{d W_{\frac{2 \mathrm{~s}}{1 \mathrm{~s}}(\overline{\mathrm{H}})}^{(1 \gamma)}}{d W_{2 \mathrm{~s} 1 \mathrm{~s}}^{(1 \gamma)}}=\frac{W_{0}\left(D_{\max }\right) 2 \beta\left(D_{\max }\right) \mathbf{n}_{\mathbf{D}} \mathbf{n}_{\mathbf{k}}}{\frac{3}{8 \pi} W_{2 \mathrm{~s} 1 \mathrm{~s}}^{(1 \gamma)}} \approx \frac{1}{5} \mathbf{n}_{\mathbf{D}} \mathbf{n}_{\mathbf{k}} .
$$

In the presence of such a very weak field given by Eq. (25), this difference is close to about $20 \%$ and probably can be observed in experiments of the type reported in [4, 5].

We would also note that if one integrates in Eq. (22) over photon emission directions, the term linear with respect to the field vanishes. But a quadratic term included in $W_{0}$ exists and represents a correction to the $W_{2 \mathrm{~s} 1 \mathrm{~s}}^{(1 \gamma)}$ transition probability, i.e. to the lifetime of the $2 \mathrm{~s}$ level. This correction term reaches the magnitude of the radiative correction obtained in [12] in the field of the strength

$$
D_{\mathrm{r}}=\frac{1}{|e|} \sqrt{\frac{8 \pi}{3} \Delta \frac{\delta \Gamma_{2 \mathrm{~s}}}{W_{2 \mathrm{p} 1 \mathrm{~s}}^{(1 \gamma)}}} \approx \sqrt{\frac{8 \pi}{3} \frac{\delta \Gamma_{2 \mathrm{~s}}}{\Gamma_{2 \mathrm{~s}}}} D_{\max }
$$

where the correction $\delta \Gamma_{2 \mathrm{~s}} / \Gamma_{2 \mathrm{~s}}$ was derived by Jentschura (see Eq. (36) in [12]). Though the correction in [12] is obtained for the process of the two-photon decay of $2 \mathrm{~s}$ level, it also represents a correction to the lifetime of the $2 \mathrm{~s}$ level. For the hydrogen atom $D_{\mathrm{r}} \sim 1.4 \cdot 10^{-7} \mathrm{~V} / \mathrm{cm}$. Since the experiments deal with differential cross sections, we compare also the linear term with the radiative correction. The linear term $\beta(D)$ reaches the magnitude of $\delta \Gamma_{2 \mathrm{~s}} / \Gamma_{2 \mathrm{~s}}$ at the field strength $7.5 \cdot 10^{-11} \mathrm{~V} / \mathrm{cm}$. It should be difficult to eliminate spurious fields of such magnitude in real experiments and, therefore, the comparison of the theoretical results in [12] with experimental ones requires some caution.

\section{TRANSITION PROBABILITIES IN DIFFERENT FORMS AND GAUGES}

In this section different gauges in combination with different "forms" for the one-photon transition probability are described. Atomic units $\hbar=e=m=1$ will be used throughout this section.

The transition probability for the emission of a photon with definite angular momentum and parity can be described in the first order of QED perturbation theory within arbitrary gauge as

$$
\begin{aligned}
W_{A \rightarrow A^{\prime}}(\omega)= & \sum_{k q}\left[\left|\left\langle A^{\prime}\left|\left(\alpha_{\mathrm{e}} \mathbf{A}_{\omega k q}(\mathbf{r})\right)+\Phi_{\omega k q}(\mathbf{r})\right| A\right\rangle\right|^{2}\right. \\
& \left.+\left|\left\langle A^{\prime}\left|\alpha_{\mathrm{m}} \mathbf{A}_{\omega k q}(\mathbf{r})\right| A\right\rangle\right|^{2}\right]
\end{aligned}
$$

where ${ }_{\mathrm{e}} \mathbf{A}_{\omega k q}$ and ${ }_{\mathrm{m}} \mathbf{A}_{\omega k q}$ denote the electric and magnetic vector potentials and $\Phi_{\omega k q}$ corresponds to the scalar potential; $\omega$ is the photon frequency, $k, q$ are the total angular momentum of the emitted photon and its projection. The bra-kets $|A\rangle$ and 
$\left\langle A^{\prime}\right|$ are stationary Dirac states (wave functions) with energies $E_{A}$ and $E_{A^{\prime}}$ and $\alpha$ are the Dirac matrices. In the momentum representation these potentials take the form

$$
\begin{aligned}
{ }_{\mathrm{e}} \mathbf{A}_{\omega k q}(\mathbf{k}) & =\frac{4 \pi^{2} c^{3 / 2}}{\omega^{3 / 2}} \delta\left(k-\frac{\omega}{c}\right)\left({ }_{\mathrm{e}} \mathbf{Y}_{k q}\left(\mathbf{n}_{\mathbf{k}}\right)+K \mathbf{n}_{\mathbf{k}} Y_{k q}\left(\mathbf{n}_{\mathbf{k}}\right)\right) \\
{ }_{\mathrm{m}} \mathbf{A}_{\omega k q}(\mathbf{k}) & =\frac{4 \pi^{2} c^{3 / 2}}{\omega^{3 / 2}} \delta\left(k-\frac{\omega}{c}\right) \mathrm{m}_{\mathrm{m}} \mathbf{Y}_{k q}\left(\mathbf{n}_{\mathbf{k}}\right), \\
\Phi_{\omega k q}(k) & =\frac{4 \pi^{2} c^{3 / 2}}{\omega^{3 / 2}} \delta\left(k-\frac{\omega}{c}\right) K Y_{k q}\left(\mathbf{n}_{\mathbf{k}}\right) .
\end{aligned}
$$

Here $\mathbf{k}$ denotes the variable in the momentum representation. Functions ${ }_{\mathrm{e}} \mathbf{Y}_{k q}$ and ${ }_{\mathrm{m}} \mathbf{Y}_{k q}$ are the vector spherical harmonics of electric and magnetic type, respectively, $Y_{k q}$ is the ordinary spherical harmonic, $c$ is the speed of light and $K$ denotes a gauge-dependent constant.

The spherical components of the transversal electric ${ }_{\mathrm{e}} A^{(1 \lambda)}$ and the longitudinal ${ }_{1} A^{(1 \lambda)}$ parts of the electromagnetic vector potential (the superscript $(1 \lambda)$ defines the rank of a spherical tensor and labels the components) are

$$
\begin{aligned}
& { }_{\mathrm{e}} A_{\omega k q}^{(1 \lambda)}=\sqrt{\frac{\omega}{\pi c(2 k+1)}}\left[\sqrt{k(2 k+3)}\left(\begin{array}{ccc}
1 & k+1 & k \\
\lambda & q-\lambda & q
\end{array}\right) g_{k+1}(\omega r) C_{-q+\lambda}^{(k+1)}\right.
\end{aligned}
$$

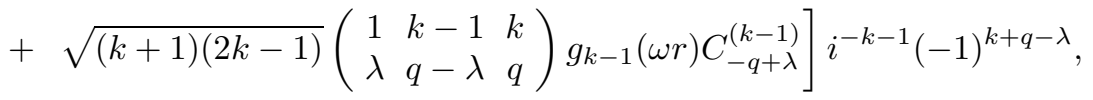

$$
\begin{aligned}
& { }_{1} A_{\omega k q}^{(1 \lambda)}=\sqrt{\frac{\omega}{\pi c(2 k+1)}}\left[\sqrt{(k+1)(2 k+3)}\left(\begin{array}{ccc}
1 & k+1 & k \\
\lambda & q-\lambda & q
\end{array}\right) g_{k+1}(\omega r) C_{-q+\lambda}^{(k+1)}\right. \\
& \left.+\sqrt{k(2 k-1)}\left(\begin{array}{ccc}
1 & k-1 & k \\
\lambda & q-\lambda & q
\end{array}\right) g_{k-1}(\omega r) C_{-q+\lambda}^{(k-1)}\right] i^{-k-1}(-1)^{k+q+\lambda+1},
\end{aligned}
$$

where $C_{-q}^{(k)}=\sqrt{\frac{4 \pi}{2 k+1}} Y_{-q}^{(k)}$ and usual notations for $3 j$-symbols are employed. The spherical components of the transverse magnetic vector potential look as

$$
{ }_{\mathrm{m}} A_{\omega q k}^{(1 \lambda)}=(-1)^{\lambda+k+q} i^{-k} \sqrt{\frac{\omega(2 k+1)}{\pi c}} g_{k}(\omega r)\left(\begin{array}{ccc}
1 & k & k \\
-\lambda & -q+\lambda & -q
\end{array}\right) C_{-q+\lambda}^{(k)}
$$

while the spherical components of the scalar potential are given by the following expression

$$
\Phi_{\omega k q}=i^{-k}(-1)^{k+q} 2 \sqrt{\frac{\omega}{c}} g_{k}(\omega r) Y_{-q}^{(k)} .
$$

The radial functions $g_{k}(\omega r)$ are related to Bessel functions $J_{\mu}(z)$ via $g_{k}(z)=(2 \pi)^{3 / 2} \frac{1}{\sqrt{z}} J_{k+\frac{1}{2}}(z)$.

Usually two gauges are used: The so-called Coulomb gauge that corresponds to the vanishing longitudinal part of the vector potential and the scalar potential (i.e. $\nabla \cdot \mathbf{A}=\nabla \cdot{ }_{\mathrm{e}} \mathbf{A}=0$ and $\Phi=0$ ). This gauge is characterized by the choice of the gauge parameter $K=0$. Another convenient gauge is defined by the following value of the parameter $K=-\sqrt{\frac{k+1}{k}}$. Within this gauge, as it can be seen from Eqs. (33) and (34), the terms containing spherical functions $C_{-q}^{(k)}$ of the order $k-1$, vanish in the expression for the transition probability (29).

After some manipulations the expression for the probability of emission of an electric photon with the angular momentum $k$ can be cast into the form

$$
W_{A \rightarrow A^{\prime}}^{\mathrm{E} k}=\frac{2(k+1) \omega}{k(2 k+1) c} \sum_{q=-k}^{k}\left|\left\langle A^{\prime}\left|\left[{ }_{\mathrm{e}} O_{-q}^{\prime(k)}+K \sqrt{\frac{k}{k+1}}\left({ }_{1} O_{-q}^{(k)}+{ }_{\Phi} O_{-q}^{(k)}\right)\right]\right| A\right\rangle\right|^{2} .
$$


Here

$$
\begin{aligned}
{ }_{\mathrm{e}} O_{-q}^{\prime(k)}= & -i\left[k \sqrt{\frac{2 k+3}{k+1}} g_{k+1}(\omega r)\left[C^{(k+1)} \times \alpha^{(1)}\right]_{-q}^{(k)}\right. \\
& \left.+\sqrt{k(2 k-1)} g_{k-1}(\omega r)\left[C^{(k-1)} \times \alpha^{(1)}\right]_{-q}^{(k)}\right], \\
{ }_{1} O_{-q}^{(k)}= & i\left[\sqrt{(k+1)(2 k+3)} g_{k+1}(\omega r)\left[C^{(k+1)} \times \alpha^{(1)}\right]_{-q}^{(k)}\right. \\
& \left.-\sqrt{k(2 k-1)} g_{k-1}(\omega r)\left[C^{(k-1)} \times \alpha^{(1)}\right]_{-q}^{(k)}\right], \\
{ }_{\Phi} O_{-q}^{(k)}= & \sqrt{2 k+1} g_{k}(\omega r) C_{-q}^{(k)},
\end{aligned}
$$

and $\left[a^{\left(s_{1}\right)} \times b^{\left(s_{2}\right)}\right]_{q}^{(s)}$ represents the tensor product of two irreducible spherical tensors of rank $s_{1}$ and $s_{2}$ coupled to a spherical tensor of rank $s$ with components $q$.

Using the following integral relation for the Dirac wave functions [37]

$$
i \int \psi_{A^{\prime}}^{*}(\alpha \nabla \chi) \psi_{A} d^{3} \tau=\frac{\omega}{c} \int \psi_{A^{\prime}}^{*} \chi \psi_{A} d^{3} \tau
$$

where $\chi$ is an arbitrary function, one can establish another form for the Ek-transition probability (see [25]):

$$
W_{A \rightarrow A^{\prime}}^{\mathrm{E} k}=\frac{2(k+1) \omega^{3}}{k(2 k+1) c^{3}} \sum_{q=-k}^{k}\left|\left\langle A^{\prime}\left|{ }_{\mathrm{e}} O_{-q}^{(k)}+K \frac{c}{\omega} \sqrt{\frac{k}{k+1}}\left[{ }_{1} O_{-q}^{(k)}+{ }_{\Phi} O_{-q}^{(k)}\right]\right| A\right\rangle\right|^{2},
$$

where

$$
\begin{aligned}
{ }_{\mathrm{e}} O_{-q}^{(k)}= & -r g_{k}(\omega r) C_{-q}^{(k)}-i \frac{r}{k+1} g_{k}(\omega r)\left[\sqrt{k(2 k-1)}\left[C^{(k-1)} \times \alpha^{(1)}\right]_{-q}^{(k)}\right. \\
& \left.+\sqrt{(k+1)(2 k+3)}\left[C^{(k-1)} \times \alpha^{(1)}\right]_{-q}^{(k)}\right]
\end{aligned}
$$

Thus, we have two different (equivalent) forms for the Ek-transition probabilities (Eqs. (37) and (42) together with an arbitrary choice for the gauge constant $K$ at our disposal. Analogous expressions for the emission probability of a photon, characterized by its energy and polarization, are provided in [40].

The aim of the present investigation concerns the derivation of the nonrelativistic limit of the E1M1-, E1E1- and E1E2transition probabilities in different gauges and forms. Deriving the nonrelativistic limit of Eqs. (37) and (42), implies two distinct nonrelativistic forms for the one-photon transition probability with arbitrary gauge constant $K$ (see [41]):

$$
W_{A \rightarrow A^{\prime}}^{\mathrm{E} k}=\frac{2(k+1)(2 k+1) \omega^{2 k-1}}{k[(2 k+1) ! !]^{2} c^{2 k+1}} \sum_{q=-k}^{k}\left|\left(A^{\prime}\left|\left(Q_{-q}^{\prime(k)}+K \sqrt{\frac{k}{k+1}}\left[Q_{-q}^{\prime(k)}-\omega Q_{-q}^{(k)}\right]\right)\right| A\right)\right|^{2}
$$

and

$$
W_{A \rightarrow A^{\prime}}^{\mathrm{E} k}=\frac{2(k+1)(2 k+1)}{k[(2 k+1) ! !]^{2}} \sum_{q=-k}^{k}\left(\frac{\omega}{c}\right)^{2 k+1}\left|\left(A^{\prime}\left|\left(Q_{-q}^{(k)}+K \sqrt{\frac{k}{k+1}}\left[\frac{1}{\omega} Q_{-q}^{(k)}-Q_{-q}^{(k)}\right]\right)\right| A\right)\right|^{2}
$$

Here $\mid A)$ and $\left(A^{\prime} \mid\right.$ are nonrelativistic Schrödinger states (wave functions) together with operators

$$
\begin{gathered}
Q_{-q}^{(k)}=-r^{k} C_{-q}^{(k)} \\
Q_{-q}^{\prime(k)}=-r^{-k-1}\left(k C_{-q}^{(k)} \frac{\partial}{\partial r}+\frac{i}{r} \sqrt{k(k+1)}\left[C^{(k)} \times L^{(1)}\right]_{-q}^{(k)}\right)
\end{gathered}
$$


where $L^{(1)}$ is the orbital angular momentum of the atomic electron. Choosing $K=0$, we find that the operator in Eq. (44) corresponds to the nonrelativistic transition operator in the "velocity" form, while for $K=-\sqrt{\frac{k+1}{k}}$ it is related to the transition operator in the "length" form. However, the correspondence of a certain gauge choice to a particular type of nonrelativistic transition operators is not unique. In view of Eq. (45) we can conclude, that within the nonrelativistic limit the expression Eq. (42) with $K=0$ converts the transition operator into the "length" form and with $K=-\sqrt{\frac{k+1}{k}}$ into the "velocity" form, respectively.

\section{APPLICATION OF THE COULOMB GREEN FUNCTION}

In order to evaluate the transition probabilities for the processes $2 \mathrm{p} \rightarrow 2 \gamma+1 \mathrm{~s}$ and $2 \mathrm{~s} \rightarrow 2 \gamma+1 \mathrm{~s}$ in the hydrogen atom the nonrelativistic Coulomb Green function is employed. The summations over the entire spectrum of the Schrödinger equation arise usually when perturbation theory is applied. The Green function approach allows one to express the intermediate summations in a closed analytic form. This is very useful for the analysis and for tests of numerical calculations.

The Green function for the Schrödinger equation with the Hamiltonian $\hat{H}$ is defined by the solution of the equation

$$
(\hat{H}-E) G\left(E ; \mathbf{r}, \mathbf{r}^{\prime}\right)=\delta\left(\mathbf{r}-\mathbf{r}^{\prime}\right)
$$

and can be always represented in terms of a spectral decomposition

$$
G\left(E ; \mathbf{r}, \mathbf{r}^{\prime}\right)=\sum_{N} \frac{\varphi_{N}^{*}(\mathbf{r}) \varphi_{N}\left(\mathbf{r}^{\prime}\right)}{E_{N}-E} .
$$

In Eq. (49) the sum runs over the entire spectrum of the Hamiltonian (bound and continuous spectrum). The set of quantum numbers $N$ may be specified as usual by the principal quantum number $n$, orbital angular momentum number $l$ and projection $m$. In view of the spherical symmetry it is sufficient to derive a closed expression for the radial part $g_{l}\left(E ; r, r^{\prime}\right)$ of the Green function defined by the partial wave decomposition

$$
G\left(E ; \mathbf{r}, \mathbf{r}^{\prime}\right)=\sum_{l m} \frac{1}{r r^{\prime}} g_{l}\left(E ; r, r^{\prime}\right) Y_{l m}^{*}\left(\mathbf{n}_{\mathbf{r}}\right) Y_{l m}\left(\mathbf{n}_{\mathbf{r}^{\prime}}\right) .
$$

In the particular case of an external Coulomb potential the Green function of Eq. (48) is called Coulomb Green Function (CGF). With the use of the expansion Eq. (50) the radial integrals occurring in Eqs. (44) and (45) for the transition probabilities can be calculated explicitly (see Ref. [42] for details).

For the radial part of the Coulomb Green function it is convenient to employ the Sturmian expansion [18], which is defined in the entire complex energy plane via

$$
\frac{1}{r r^{\prime}} g_{l}\left(E ; r, r^{\prime}\right)=\sum_{n_{r}=0}^{\infty} \frac{\Phi_{n_{r} l}(r) \Phi_{n_{r} l}\left(r^{\prime}\right)}{E_{n_{r} l}-E}
$$

where $\Phi_{n_{r} l}(r)$ denote the Sturmian functions. The Sturmian expansion of the CGF can be represented in an alternative form by introducing radial functions

$$
R_{n_{r} l}\left(\frac{2 r}{\nu}\right)=\frac{1}{r} \sqrt{\frac{Z}{\nu n_{r}}} \frac{1}{(2 l+1) !} \sqrt{\frac{\Gamma\left(n_{r}+l+1\right)}{\Gamma\left(n_{r}-l\right)}} M_{n_{r}, l+\frac{1}{2}}\left(\frac{2 r}{\nu}\right),
$$

which are related to Whittaker functions $M_{n_{r}, l+\frac{1}{2}}\left(\frac{2 r}{\nu}\right)$, where $\nu=Z / \sqrt{-2 E}$. For integer values $\nu=n$ these functions coincide with the normalized, radial hydrogenic wave functions

$$
\Phi_{n_{r} l}(r)=\sqrt{\frac{\nu n}{Z}} R_{n l}\left(\frac{2 r}{\nu}\right)
$$

Substitution of Eq. (53) into (51) yields

$$
\frac{1}{r r^{\prime}} g_{l}\left(\nu ; r, r^{\prime}\right)=\frac{\nu^{2}}{Z^{2}} \sum_{n=l+1}^{\infty} \frac{n}{n-\nu} R_{n l}\left(\frac{2 r}{\nu}\right) R_{n l}\left(\frac{2 r^{\prime}}{\nu}\right) .
$$


Within this paper we apply the Green function method for the evaluation of the two-photon decay probability in the hydrogen atom.

In [43] the two-photon transition process $2 \mathrm{~s} \rightarrow \gamma(\mathrm{E} 1)+\gamma(\mathrm{E} 1)+1$ s has been considered. The probability for the two-photon decay $A \rightarrow \gamma(\mathrm{E} 1)+\gamma(\mathrm{E} 1)+A^{\prime}$ with photon frequencies $\omega_{1}$ and $\omega_{2}$ within the nonrelativistic limit and dipole approximation is:

$$
\begin{aligned}
d W_{A \rightarrow A^{\prime}}^{\mathrm{E} 1 \mathrm{E} 1}\left(\omega_{2}\right)= & \frac{8}{9 \pi}\left(\frac{4 \pi}{3}\right)^{3} \sum_{M_{1} M_{2}} \mid\left(A^{\prime}\left|r Y_{1 M_{2}}\left(\mathbf{n}_{\mathbf{r}}\right) G\left(E_{A}-\omega_{1} ; \mathbf{r}, \mathbf{r}^{\prime}\right) r^{\prime} Y_{1 M_{1}}^{*}\left(\mathbf{n}_{\mathbf{r}^{\prime}}\right)\right| A\right) \\
& +\left.\left(A^{\prime}\left|r Y_{1 M_{1}}\left(\mathbf{n}_{\mathbf{r}}\right) G\left(E_{A}-\omega_{2} ; \mathbf{r}, \mathbf{r}^{\prime}\right) r^{\prime} Y_{1 M_{2}}^{*}\left(\mathbf{n}_{\mathbf{r}^{\prime}}\right)\right| A\right)\right|^{2}\left(\omega_{1} \omega_{2}\right)^{2} d \omega_{2} .
\end{aligned}
$$

The energy conservation law implies $\omega_{1}=E_{A}-E_{A^{\prime}}-\omega_{2}$. After the evaluation of angular matrix elements in Eq. (55) the remaining radial integrals have the form

$$
\begin{array}{r}
\int_{0}^{\infty} \int_{0}^{\infty} \int_{0}^{\infty} d r^{\prime} d r d x\left(r^{\prime}\right)^{s^{\prime}+\frac{7}{2}}(r)^{s+\frac{7}{2}} \exp \left(-\frac{1}{\nu}\left(\beta^{\prime} r^{\prime}+\beta r\right)+\left(r+r^{\prime}\right) \cosh (x)\right) \\
\times\left(\operatorname{coth}\left(\frac{x}{2}\right)\right)^{2 \nu} I_{2 l+1}\left(\frac{2 \sqrt{r r^{\prime}}}{\nu} \sinh (x)\right),
\end{array}
$$

where $n$ and $n^{\prime}$ are the principal quantum numbers of the initial and final states, respectively, together with the parameters $\beta=\nu / n, \beta^{\prime}=\nu / n^{\prime}$ and $\nu=\sqrt{-2\left(E_{n l}-\omega\right)}$. These integrals can be evaluated analytically after inserting the series expansion for the modified Bessel functions $I_{2 l+1}$. The integration over $x$ should be done at the end. Assuming emission of two E1 photons, one can write the total probability for such a two-photon decay as

$$
W_{A \rightarrow A^{\prime}}^{2 \mathrm{E} 1}=\frac{1}{2} \int_{0}^{\omega_{0}} d W_{A \rightarrow A^{\prime}}^{2 \mathrm{E} 1}(\omega)
$$

with $\omega_{0}=E_{A}-E_{A^{\prime}}$. For the process $2 \mathrm{~s} \rightarrow 2 \gamma(\mathrm{E} 1)+1 \mathrm{~s}$ in [43] the result of the evaluations was reported as

$$
W_{2 \mathrm{~s} 1 \mathrm{~s}}^{2 E 1}=8.226(\alpha Z)^{6} \mathrm{~s}^{-1}
$$

with an accuracy of about $1 \%$.

However, in further calculations it is convenient to utilize the other representation of the radial Coulomb Green function in terms of an expansion over Laguerre polynomials [18] (employed also in Ref. [43])

$$
g_{l}\left(\nu ; r, r^{\prime}\right)=\frac{4 Z}{\nu}\left(\frac{4}{\nu^{2}} r r^{\prime}\right)^{l} \exp \left(-\frac{r+r^{\prime}}{\nu}\right) \sum_{n=0}^{\infty} \frac{n ! L_{n}^{2 l+1}\left(\frac{2 r}{\nu}\right) L_{n}^{2 l+1}\left(\frac{2 r^{\prime}}{\nu}\right)}{(2 l+1+n) !(n+l+1-\nu)} .
$$

The series Eq. (59) converges absolutely as $n^{-3 / 2}$ for arguments $r, r^{\prime}>0$ and $\operatorname{Im}(\nu)=0$ [18]. The angular momentum quantum number $l=1$ for the intermediate states is fixed after the angular integration. Inserting the expansion (59) for $l=1$ into the expression (55) yields

$$
\begin{gathered}
d W_{2 \mathrm{~s} 1 \mathrm{~s}}^{2 \mathrm{E} 1}(\omega)=\left(\frac{2}{3}\right)^{3} \frac{\omega^{3} \omega^{\prime 3}}{\pi}\left[I_{1}(\nu)+I_{1}\left(\nu^{\prime}\right)\right]^{2} \alpha^{6} d \omega, \\
I_{1}(\nu)=\frac{16 \sqrt{2}}{\nu^{3}}\left(\frac{\nu}{2}\right)^{10} \sum_{m=0}^{\infty} \frac{m !}{(m+3) !(m+2-\nu)} \int_{0}^{\infty} d \xi \xi^{4} e^{-\xi\left(\frac{\nu+1}{2}\right)} L_{m}^{3}(\xi) \\
\times \int_{0}^{\infty} d t t^{4} e^{-t\left(\frac{\nu+2}{4}\right)}\left(1-\frac{\nu}{4} t\right) L_{m}^{3}(t) .
\end{gathered}
$$

These integrals can be evaluated analytically. After inserting this result into Eq. (57) the integration over $\omega$ has to be performed in order to obtain the total transition probability for the E1E1 decay of the $2 \mathrm{~s}$ state in the hydrogen atom. This is achieved 
numerically with the aid of the computer-algebra code MATHEMATICA. The final result is

$$
W_{2 \mathrm{~s} 1 \mathrm{~s}}^{2 \mathrm{E} 1}=\frac{1}{2} \int_{0}^{\omega_{0}} d W_{2 \mathrm{~s} 1 \mathrm{~s}}^{2 \mathrm{E} 1}(\omega)=0.00131823(\alpha Z)^{6} \text { a.u. }=8.22932 \mathrm{~s}^{-1}(Z=1)
$$

with $\omega_{0}=E_{2 \mathrm{~s}}-E_{1 \mathrm{~s}}$. In Eq. (62) we indicated the Z-dependence of the $W_{2 \mathrm{~s} 1 \mathrm{~s}}^{\mathrm{E} 1 \mathrm{E} 1}$ transition probability. The numerical value (62) coincides with most precise result [12] up to 5 digits. This will serve us as accuracy estimate of our approach in following calculations.

\section{E1E2 DECAY PROBABILITY FOR THE 2P STATE}

In this section we consider the E1E2 decay of the 2p state in the hydrogen atom. Again the set of quantum numbers $n l m_{l}$ is employed as far as the total angular momentum $j$ is not important in this calculation performed within the nonrelativistic approach. Nevertheless, in order to compare our results with those obtained from the relativistic evaluation (see Refs. [14], [15]), we shall perform the calculation within two different gauges according to Eqs. (44)-(47). This will also elucidate the potential influence of relativistic effects associated with the contribution of the negative-energy Dirac spectrum.

As a test for the method the gauge constant $K=-\sqrt{\frac{k+1}{k}}$ is chosen in the expression Eq. (44) for the transition probability, which corresponds to the nonrelativistic "length" form as mentioned above. This would be equivalent to the choice $K=0$ together with the form Eq. (45). Inspection of Eqs. (44) and (46) reveals that the emission of electric photons (Ek) is described by the potentials

$$
V^{\mathrm{E} k}=\sqrt{\frac{k+1}{k}} \frac{2 \omega^{k+\frac{1}{2}}}{(2 k+1) ! !} r^{k} Y_{k-q} .
$$

Accordingly, the two electric photon decay rate of the atomic state $A$ can be written as

$$
d W_{A \rightarrow A^{\prime}}^{\mathrm{E} k \mathrm{E} k^{\prime}}=\sum_{q q^{\prime} m_{A} m_{A^{\prime}}}\left|\sum_{N} \frac{\left(A^{\prime}\left|V^{\mathrm{E} k}\right| N\right)\left(N\left|V^{\mathrm{E} k^{\prime}}\right| A\right)}{E_{N}-E_{A}+\omega}+\sum_{N} \frac{\left(A^{\prime}\left|V^{\mathrm{E} k^{\prime}}\right| N\right)\left(N\left|V^{\mathrm{E} k}\right| A\right)}{E_{N}-E_{A}+\omega^{\prime}}\right|^{2} \delta\left(\omega+\omega^{\prime}-E_{A}+E_{A^{\prime}}\right) d \omega d \omega^{\prime} .
$$

Here the labels $A, A^{\prime}$ and $N$ abbreviate the set of nonrelativistic quantum numbers (principal quantum number $n$, orbital momentum $l$ and projection $m_{l}$ ) for indicating the state of the atomic electron as the initial $(A)$, intermediate $(N)$ and final $\left(A^{\prime}\right)$. The photons will be characterized by the angular momentum and its projection $(k q)$ as well as by the type of the photon (electric or magnetic). The Eq. (64) also implies the summation over degenerate substates of the final atomic state $A^{\prime}$ and the average over the degenerate substates of the initial atomic state $A$ as well as summations over the angular momentum projections of both emitted photons. The frequencies of the two photons $\omega$ and $\omega^{\prime}$ are related by the energy conservation law $\omega^{\prime}=\omega_{0}-\omega$, where $\omega_{0}=E_{A}-E_{A^{\prime}}$.

Employing the eigenmode decomposition of the Coulomb Green function Eq. (50) the probability of the two-photon decay process takes the form

$$
\begin{array}{r}
d W_{A \rightarrow A^{\prime}}^{\mathrm{E} k \mathrm{E} k^{\prime}}=\frac{2 \pi}{2 l_{A}+1} \sum_{q q^{\prime} m_{A} m_{A^{\prime}}} \mid \sum_{l m_{l}} \iint d \mathbf{r}_{\mathbf{1}} d \mathbf{r}_{\mathbf{2}} R_{n_{A^{\prime}} l_{A^{\prime}}}\left(r_{1}\right) Y_{l_{A^{\prime}} m_{A^{\prime}}}^{*}\left(\mathbf{n}_{\mathbf{r}_{\mathbf{1}}}\right) \\
\times V^{\mathrm{E} k}\left(\mathbf{r}_{\mathbf{1}}\right) g_{l}\left(\nu ; r_{1}, r_{2}\right) Y_{l m_{l}}\left(\mathbf{n}_{\mathbf{r}_{1}}\right) Y_{l m_{l}}^{*}\left(\mathbf{n}_{\mathbf{r}_{\mathbf{2}}}\right) V^{\mathrm{E} k^{\prime}}\left(\mathbf{r}_{\mathbf{2}}\right) R_{n_{A} l_{A}}\left(r_{2}\right) Y_{l_{A} m_{A}}\left(\mathbf{n}_{\mathbf{r}_{\mathbf{2}}}\right)+ \\
+\sum_{l m_{l}} \iint d \mathbf{r}_{\mathbf{1}} d \mathbf{r}_{\mathbf{2}} R_{n_{A^{\prime}} l_{A^{\prime}}}\left(r_{1}\right) Y_{l_{A^{\prime}} m_{A^{\prime}}}^{*}\left(\mathbf{n}_{\mathbf{r}_{\mathbf{1}}}\right) V^{\mathrm{E} k^{\prime}}\left(\mathbf{r}_{\mathbf{1}}\right) g_{l}\left(\nu^{\prime} ; r_{1}, r_{2}\right) Y_{l m_{l}}\left(\mathbf{n}_{\mathbf{r}_{1}}\right) \\
\times\left. Y_{l m_{l}}^{*}\left(\mathbf{n}_{\mathbf{r}_{\mathbf{2}}}\right) V^{\mathrm{E} k}\left(\mathbf{r}_{\mathbf{2}}\right) R_{n_{A} l_{A}}\left(r_{2}\right) Y_{l_{A} m_{A}}\left(\mathbf{n}_{\mathbf{r}_{\mathbf{2}}}\right)\right|^{2} d \omega,
\end{array}
$$

where $V^{\mathrm{E} k}(\mathbf{r})$ is the potential (63) written in the gauge $K=-\sqrt{\frac{k+1}{k}}$ and compatible with the form Eq. (44), together with parameters $\nu=Z / \sqrt{-2\left(E_{A}-\omega\right)}, \nu^{\prime}=Z / \sqrt{-2\left(E_{A}-\omega^{\prime}\right)}$ and frequency $\omega^{\prime}=E_{A}-E_{A^{\prime}}-\omega$.

Specifying Eq. 65) for the transition between levels $A=2 \mathrm{p}, A^{\prime}=1 \mathrm{~s}$ and taking into account that in this case the angular momentum of the photon can take values $k=1,2$, we receive four different terms contributing in Eq. (65). Unfortunately, in 
the previous paper [24] an error in the summation over projections of the Clebsch-Gordan coefficients did occur (see expression Eq. (41) in [24]). Here we correct this mistake and give the proper expression for the probability. After angular integration and summation over projections we find

$$
d W_{2 \mathrm{p} 1 \mathrm{~s}}^{\mathrm{E} 1 \mathrm{E} 2}(\omega)=\frac{2^{2} \omega^{3} \omega^{\prime 3}}{3^{3} 5^{2} \pi}\left[\omega^{\prime 2}\left|I_{1}\left(\omega^{\prime}\right)+I_{2}(\omega)\right|^{2}+\omega^{2}\left|I_{1}(\omega)+I_{2}\left(\omega^{\prime}\right)\right|^{2}\right] d \omega,
$$

where

$$
I_{1}(\omega)=\frac{1}{\sqrt{6}} \int_{0}^{\infty} \int_{0}^{\infty} d r_{1} d r_{2} r_{1}^{3} r_{2}^{5} e^{-r_{1}-\frac{r_{2}}{2}} g_{1}\left(E_{A}-\omega ; r_{1}, r_{2}\right)
$$

and

$$
I_{2}(\omega)=\frac{1}{\sqrt{6}} \int_{0}^{\infty} \int_{0}^{\infty} d r_{1} d r_{2} r_{1}^{4} r_{2}^{4} e^{-r_{1}-\frac{r_{2}}{2}} g_{2}\left(E_{A}-\omega ; r_{1}, r_{2}\right)
$$

respectively. Inserting again the representation Eq. (59) for the CGF with $l=1$ leads to radial integrals that can be evaluated analytically.

Substituting the integrals (67) and (68) into (66) and integrating over frequencies $\omega$ yields

$$
W_{2 \mathrm{p} 1 \mathrm{~s}}^{\mathrm{E} 1 \mathrm{E} 2}=\frac{1}{2} \int_{0}^{\omega_{0}} d W_{2 \mathrm{p} 1 \mathrm{~s}}^{\mathrm{E} 1 \mathrm{E} 2}=1.98896 \cdot 10^{-5}(\alpha Z)^{8} \text { a.u. }=6.61197 \cdot 10^{-6} \mathrm{~s}^{-1}(Z=1)
$$

with $\omega_{0}=E_{2 \mathrm{p}}-E_{1 \mathrm{~s}}$. In Eq. (69) we indicated the Z-dependence of the $W_{2 \mathrm{p} 1 \mathrm{~s}}^{\mathrm{E} 1 \mathrm{E} 1}$ transition probability. Compared with the relativistic result in the "length" gauge (see [15]) the relative discrepancy is about $0.1 \%$.

The calculation of the E1E2 two-photon decay with the nonrelativistic "velocity" form is more involved. Now the gauge constant should be chosen either $K=-\sqrt{\frac{k+1}{k}}$ for the form Eq. (45) or $K=0$ for the form Eq. (44).

We choose $K=0$ together with the form Eq. (44). The potential in this case reads

$$
V^{\mathrm{E} k}(\mathbf{r})=\frac{4 \omega^{k-\frac{1}{2}}}{(2 k+1) ! !} \sqrt{\frac{k+1}{k(2 k+1)}} r^{k-1}\left[k Y_{-q}^{(k)}\left(\mathbf{n}_{\mathbf{r}}\right) \frac{\partial}{\partial r}+\frac{i}{r} \sqrt{k(k+1)}\left[Y^{(k)} \times L^{(1)}\right]_{-q}^{k}\right] .
$$

The formula for $d W_{2 \mathrm{p} 1 \mathrm{~s}}^{\mathrm{E} 1 \mathrm{E}}$ follows again from Eq. (65). Performing angular integrations and summations over projections as discussed in previous cases now yields

$$
d W_{2 \mathrm{p} 1 \mathrm{~s}}^{\mathrm{E} 1 \mathrm{E} 2}(\omega)=\frac{2^{4}}{3^{3} 5^{2} \pi} \omega^{\prime} \omega\left[\omega^{2}\left|I_{1}(\omega)+I_{2}\left(\omega^{\prime}\right)\right|^{2}+\omega^{\prime 2}\left|I_{1}\left(\omega^{\prime}\right)+I_{2}(\omega)\right|^{2}\right] d \omega
$$

with radial integrals of the type

$$
I_{1}(\omega)=\frac{1}{\sqrt{6}} \int_{0}^{\infty} \int_{0}^{\infty} d r_{1} d r_{2} r_{1}^{2} r_{2}^{3} e^{-r_{1}-\frac{r_{2}}{2}}\left[1-\frac{9 i}{2}-\frac{r_{2}}{2}\right]\left[\frac{\partial}{\partial r_{1}}-\frac{2 i}{r_{1}}\right] g_{1}\left(E_{A}-\omega ; r_{1}, r_{2}\right)
$$

and

$$
I_{2}(\omega)=\frac{1}{\sqrt{6}} \int_{0}^{\infty} \int_{0}^{\infty} d r_{1} d r_{2} r_{1}^{3} r_{2}^{2} e^{-r_{1}-\frac{r_{2}}{2}}\left[1-5 i-\frac{r_{2}}{2}\right]\left[\frac{\partial}{\partial r_{1}}-\frac{3 i}{r_{1}}\right] g_{2}\left(E_{A}-\omega ; r_{1}, r_{2}\right)
$$

together with parameters $\nu=Z / \sqrt{-2\left(E_{2 \mathrm{p}}-\omega\right)}$ and $\nu^{\prime}=Z / \sqrt{-2\left(E_{2 \mathrm{p}}-\omega^{\prime}\right)}$, respectively. The integrations over $r_{1}$ and $r_{2}$ lead to a rather lengthy analytical expression containing various combinations of notations similar to those in Eqs. 67) and (68). The numerical evaluation yields finally

$$
W_{2 \mathrm{p} 1 \mathrm{~s}}^{\mathrm{E} 1 \mathrm{E} 2}=\frac{1}{2} \int_{0}^{\omega_{0}} d W_{2 \mathrm{p} 1 \mathrm{~s}}^{\mathrm{E} 1 \mathrm{E} 2}(\omega)=3.6896 \cdot 10^{-6}(\alpha Z)^{8} \text { a.u. } \simeq 1.227 \cdot 10^{-6} \mathrm{~s}^{-1}(Z=1),
$$


where $\omega_{0}=3 / 8$ a.u. This result differs from that obtained from relativistic calculations [14], [15] by about $0.5 \%$. This discrepancy is larger than the relativistic corrections (of the relative order $\alpha^{2}$ ) and can be attributed to the pure numerical uncertainties in both calculations. Note, that unlike the case of the "length" form, the negative-energy contribution is no longer negligible when the "velocity" form is employed. Therefore, the result Eq. (74) does not coincide with Eq. (69) and represents only the positive-energy contribution to $W_{2 \mathrm{p} 1 \mathrm{~s}}^{\mathrm{EE} 2}$ in the "velocity" form. Correspondingly, we compare this result to the positiveenergy contribution calculated in [14], [15]. The negative-energy contribution to $W_{2 \mathrm{p} 1 \mathrm{~s}}^{\mathrm{E} 1 \mathrm{E} 2}$ in the "velocity" form for low $Z$ values was evaluated analytically in [15].

\section{E1M1 TWO-PHOTON DECAY}

For the mixed E1M1 two-photon transition probability the expression (64) should be replaced by

$$
\begin{array}{r}
d W_{A \rightarrow A^{\prime}}^{\mathrm{E} 1 \mathrm{M} 1}=\sum_{M_{e} M_{m} m_{A} m_{A^{\prime}}} \mid \sum_{N} \frac{\left(A^{\prime}\left|V^{\mathrm{E} 1}(\omega)\right| N\right)\left(N\left|V^{\mathrm{M} 1}\left(\omega^{\prime}\right)\right| A\right)}{E_{N}-E_{A}+\omega} \\
+\frac{\left(A^{\prime}\left|V^{\mathrm{M} 1}\left(\omega^{\prime}\right)\right| N\right)\left(N\left|V^{\mathrm{E} 1}(\omega)\right| A\right)}{E_{N}-E_{A}+\omega^{\prime}} \\
+\frac{\left(A^{\prime}\left|V^{\mathrm{E} 1}\left(\omega^{\prime}\right)\right| N\right)\left(N\left|V^{\mathrm{M} 1}(\omega)\right| A\right)}{E_{N}-E_{A}+\omega^{\prime}} \\
+\left.\frac{\left(A^{\prime}\left|V^{\mathrm{M} 1}(\omega)\right| N\right)\left(N\left|V^{\mathrm{E} 1}\left(\omega^{\prime}\right)\right| A\right)}{E_{N}-E_{A}+\omega}\right|^{2} d \omega .
\end{array}
$$

Here $V^{\mathrm{E} 1}(\omega)=\frac{4}{3} \omega^{3 / 2} r Y_{M_{\mathrm{e}}}^{(1)}, V^{\mathrm{M} 1}(\omega)=\sqrt{\frac{4}{3}} \mu_{0} \omega^{3 / 2}\left(\hat{j}_{1 M_{\mathrm{m}}}+\hat{s}_{1 M_{\mathrm{m}}}\right), Y_{M_{e}}^{(1)}$ is the spherical tensor of the rank 1 with the spherical component $M_{e}$ (i.e. the spherical function $Y_{1 M_{e}}$ ), $\mu_{0}=\frac{\alpha}{2}$ is the Bohr's magneton, $\hat{j}_{1 M_{m}}$ and $\hat{s}_{1 M_{m}}$ are the spherical components of the total angular momentum and the spin operator (spherical tensors of rank 1) of the electron. This choice corresponds to the nonrelativistic "length" form for describing the emission of the electric photons. Since the potential for the magnetic photon includes total angular momentum and spin operator, coupled wave functions with the set of quantum numbers $N=\{$ nlsjm $\}$ should be used, i.e.

$$
\phi_{n l s j m}=\sum_{m_{l} m_{s}} C_{l m_{l} s m_{s}}^{j m} R_{n l}(r) Y_{m_{l}}^{(l)}\left(\mathbf{n}_{\mathbf{r}}\right) \chi_{s m_{s}}
$$

where $R_{n l}(r)$ is the solution of the radial Schrödinger equation and $\chi_{s m_{s}}(s=1 / 2)$ is the spin function. The magnetic potentials in Eq. (75) do not depend on radial variables. Thus, only the intermediate state with $n l=n_{A} l_{A}$ or $n l=n_{A^{\prime}} l_{A^{\prime}}$ will contribute to the probability in Eq. (75). After performing angular integrations and summations over all projections one arrives at the expressions

$$
d W_{2 \mathrm{p} 1 \mathrm{~s}}^{\mathrm{E} 1 \mathrm{M} 1}(\omega)=\frac{2^{8} \mu_{0}^{2}}{\pi}\left(\frac{2}{3}\right)^{12} \omega \omega^{\prime 3} d \omega
$$

and

$$
W_{2 \mathrm{p} 1 \mathrm{~s}}^{\mathrm{E} 1 \mathrm{M} 1}=\frac{1}{2} \int_{0}^{3 / 8} d W_{2 \mathrm{p} 1 \mathrm{~s}}^{\mathrm{E} 1 \mathrm{M} 1}(\omega)=\frac{2^{5}}{\pi}\left(\frac{2}{3}\right)^{12} \alpha^{8} \int_{0}^{3 / 8} \omega\left(\frac{3}{8}-\omega\right)^{3} d \omega .
$$

As the final result we obtain

$$
W_{2 \mathrm{p} 1 \mathrm{~s}}^{\mathrm{E} 1 \mathrm{M} 1}=\frac{2^{5}}{\pi}\left(\frac{2}{3}\right)^{12} \frac{243}{655360}(\alpha Z)^{8} \text { a.u. }=9.6769 \cdot 10^{-6} \mathrm{~s}^{-1}(Z=1) .
$$

Again the Z-dependence of the $W_{2 \mathrm{p} 1 \mathrm{~s}}^{\mathrm{E} 1 \mathrm{M} 1}$ transition probability is indicated. Comparison with the result of a fully relativistic calculation now reveals a discrepancy of about $0.1 \%$. 


\section{E1E2 AND E1M1 TRANSITION PROBABILITIES FOR THE 2P HYDROGENIC STATE EXPRESSED THROUGH THE PHOTON MOMENTUM AND POLARIZATION.}

In this section we are going to derive expressions which allow the analysis of the dependence on the directions and polarization degrees of freedom of the emitted photons. For this purpose we turn back to the generic $S$-matrix formulation employing the set of quantum numbers defined by polarization vector $\mathbf{e}$ and wave vector $\mathbf{k}$. Relativistic units are used throughout this section.

The $S$-matrix element of the two-photon decay process $A \rightarrow A^{\prime}+2 \gamma$ for the noninteracting electrons is represented by

$$
S_{A^{\prime} A}^{(2 \gamma)}=(-i)^{2} e^{2} \int d x_{1} d x_{2}\left(\bar{\psi}_{A^{\prime}}\left(x_{1}\right) \gamma^{\mu} A_{\mu}^{*}\left(x_{1}\right) S\left(x_{1}, x_{2}\right) \gamma^{\nu} A_{\nu}^{*}\left(x_{2}\right) \psi_{A}\left(x_{2}\right)\right),
$$

where $x_{1}=\left(\mathbf{r}_{1}, t_{1}\right)$ and $x_{2}=\left(\mathbf{r}_{2}, t_{2}\right)$ are 4-vectors. Also

$$
S\left(x_{1}, x_{2}\right)=\frac{1}{2 \pi i} \int_{-\infty}^{\infty} d \omega_{1} e^{i \omega_{1}\left(t_{1}-t_{2}\right)} \sum_{n} \frac{\psi_{n}\left(x_{1}\right) \bar{\psi}_{n}\left(x_{2}\right)}{E_{n}(1-i 0)+\omega_{1}}
$$

is the electron propagator where the sum runs over the Dirac spectrum for the electron in the field of the nucleus, $\psi_{n}(x)$ is the electron Dirac wave function, $E_{n}$ is the electron energy,

$$
A_{\mu}^{\mathbf{k}, \lambda}(x)=\sqrt{\frac{2 \pi}{\omega}} e_{\mu}^{(\lambda)} e^{i(\mathbf{k r}-\omega t)}
$$

is the wave function of the photon characterized by the momentum $\mathbf{k}$ and polarization vector $e_{\mu}^{\lambda}(\mu, \lambda=1,2,3,4), x \equiv(\mathbf{r}, t)$. For the real transverse photons

$$
\mathbf{A}(x)=\sqrt{\frac{2 \pi}{\omega}} \mathbf{e} e^{i(\mathbf{k r}-\omega t)} \equiv \sqrt{\frac{2 \pi}{\omega}} \mathbf{A}_{\mathbf{e}, \mathbf{k}}(\mathbf{r}) e^{-i \omega t} .
$$

Inserting Eqs. (81)-83) in Eq. (80), integrating over time and frequency variables and introducing the amplitude $U_{A^{\prime} A}$ as

$$
S_{A A^{\prime}}^{(2 \gamma)}=-2 \pi i \delta\left(E_{A^{\prime}}+\omega+\omega^{\prime}-E_{A}\right) U_{A^{\prime} A}^{(2 \gamma)}
$$

we obtain

$$
U_{A^{\prime} A}^{(2 \gamma)}=\frac{2 \pi e^{2}}{\sqrt{\omega \omega^{\prime}}} \sum_{n} \frac{\left(\alpha \mathbf{A}_{\mathbf{e}, \mathbf{k}}^{*}\right)_{A^{\prime} n}\left(\alpha \mathbf{A}_{\mathbf{e}^{\prime}, \mathbf{k}^{\prime}}^{*}\right)_{n A}}{E_{n}-E_{A}+\omega^{\prime}}
$$

where $e$ is the electron charge.

Taking into account photon permutation symmetry we define the transition probability as

$$
d W_{A^{\prime} A}=2 \pi \delta\left(E_{A}-E_{A^{\prime}}-\omega-\omega^{\prime}\right)\left|U_{A^{\prime} A}^{(2 \gamma) a}+U_{A^{\prime} A}^{(2 \gamma) b}\right|^{2} \frac{d \mathbf{k}}{(2 \pi)^{3}} \frac{d \mathbf{k}^{\prime}}{(2 \pi)^{3}} .
$$

Taking $d \mathbf{k} \equiv \omega^{2} d \mathbf{n}_{\mathbf{k}} d \omega$ and integrating over $\omega$ yields

$$
\begin{array}{r}
d W_{A^{\prime} A}^{(2 \gamma)}\left(\omega^{\prime}, \mathbf{n}_{\mathbf{k}}, \mathbf{n}_{\mathbf{k}^{\prime}}, \mathbf{e}, \mathbf{e}^{\prime}\right)=e^{4} \frac{\omega^{\prime}\left(E_{A}-E_{A}^{\prime}-\omega^{\prime}\right)}{(2 \pi)^{3}} \sum_{m_{A} m_{A^{\prime}}} \frac{1}{2 j_{A}+1} \\
\times\left|\sum_{n} \frac{\left(\alpha \mathbf{A}_{\mathbf{e}, \mathbf{k}}^{*}\right)_{A^{\prime} n}\left(\alpha \mathbf{A}_{\mathbf{e}^{\prime}, \mathbf{k}^{\prime}}^{*}\right)_{n A}}{E_{n}-E_{A}+\omega^{\prime}}+\sum_{n} \frac{\left(\alpha \mathbf{A}_{\mathbf{e}^{\prime}, \mathbf{k}^{\prime}}^{*}\right)_{A^{\prime} n}\left(\alpha \mathbf{A}_{\mathbf{e}, \mathbf{k}}^{*}\right)_{n A}}{E_{n}-E_{A}+\omega}\right|^{2} d \mathbf{n}_{\mathbf{k}} \mathbf{n}_{\mathbf{k}^{\prime}} d \omega^{\prime} .
\end{array}
$$

Here the summation over $n$ abbreviates the summation over the whole set of quantum numbers $n j_{n} l_{n} m_{n}$ of intermediate states. The sums over the projections of the total angular momentum of the final state $A^{\prime}$ and the averaging over the projections of the total angular momentum of the initial state $A$ in Eq. (87) are also included.

In the Pauli approximation (see Eq. (97) the (87) expression can be rewritten as

$$
\begin{array}{r}
d W_{A^{\prime} A}^{\mathrm{P}(2 \gamma)}=e^{4} \frac{\omega^{\prime}\left(E_{A}-E_{A^{\prime}}-\omega^{\prime}\right)}{(2 \pi)^{3}} \sum_{m_{A} m_{A^{\prime}}} \frac{1}{2 j_{A}+1} \\
\times\left|\sum_{n} \frac{U_{A^{\prime} n}^{\mathrm{P}}(\mathbf{e}, \mathbf{k}) U_{n A}^{\mathrm{P}}\left(\mathbf{e}^{\prime}, \mathbf{k}^{\prime}\right)}{E-n-E_{A}+\omega^{\prime}}+\sum_{n} \frac{U_{A^{\prime} n}^{\mathrm{P}}\left(\mathbf{e}^{\prime}, \mathbf{k}^{\prime}\right) U_{n A}^{\mathrm{P}}(\mathbf{e}, \mathbf{k})}{E-n-E_{A}+\omega}\right|^{2} d \mathbf{n}_{\mathbf{k}} \mathbf{n}_{\mathbf{k}^{\prime}} d \omega^{\prime}
\end{array}
$$


where $U_{A^{\prime} n}^{\mathrm{P}}(\mathbf{e}, \mathbf{k})$ is defined by Eq. (9).

Within the Pauli approximation we have to take into account the two terms of the exponent expansion in the expression for the emission operator

$$
A^{\mathrm{P}}(\mathbf{e}, \mathbf{k})=\left(\left(\mathbf{e}^{*} \hat{\mathbf{p}}\right)+i\left(\mathbf{e}^{*}[\mathbf{k} \times \hat{\mathbf{s}}]\right)\right) e^{-i \mathbf{k r}} \approx \frac{i m}{\hbar}\left[\hat{H}, \mathbf{e}^{*} \mathbf{r}\right]+\frac{m}{2 \hbar}\left[\hat{H},\left(\mathbf{e}^{*} \mathbf{r}\right)(\mathbf{k r})\right]+\frac{i}{2}\left(\mathbf{e}^{*}[\mathbf{k} \times([\mathbf{r} \times \hat{\mathbf{p}}]+2 \hat{\mathbf{s}})]\right) .
$$

The first term in this expression represents the electric dipole moment of the emitted photon, the second one is the electric quadrupole moment, and the last term in Eq. (89) represents the magnetic dipole moment of the emitted photon.

For the beginning we evaluate the two-photon E1 and E2 decay rate. The probability of this process can be written as

$$
\begin{array}{r}
d W_{A^{\prime} A}^{(\mathrm{E} 1 \mathrm{E} 2)}\left(\omega^{\prime}\right)=e^{4} \frac{\omega^{\prime}\left(E_{A}-E_{A^{\prime}}-\omega^{\prime}\right)}{(2 \pi)^{3}} \sum_{m_{A} m_{A^{\prime}}} \frac{1}{2 j_{A}+1} \\
\times \mid \sum_{n} \frac{U_{A^{\prime} n}^{\mathrm{P}(\mathrm{E} 1)}(\mathbf{e}, \mathbf{k}) U_{n A}^{\mathrm{P}(\mathrm{E} 2)}\left(\mathbf{e}^{\prime}, \mathbf{k}^{\prime}\right)+U_{A^{\prime} n}^{\mathrm{P}(\mathrm{E} 2)}(\mathbf{e}, \mathbf{k}) U_{n A}^{\mathrm{P}(\mathrm{E} 1)}\left(\mathbf{e}^{\prime}, \mathbf{k}^{\prime}\right)}{E_{n}-E_{A}+\omega^{\prime}} \\
+\left.\sum_{n} \frac{U_{A^{\prime} n}^{\mathrm{P}(\mathrm{E} 1)}\left(\mathbf{e}^{\prime}, \mathbf{k}^{\prime}\right) U_{n A}^{\mathrm{P}(\mathrm{E} 2)}(\mathbf{e}, \mathbf{k})+U_{A^{\prime} n}^{\mathrm{P}(\mathrm{E} 2)}\left(\mathbf{e}^{\prime}, \mathbf{k}^{\prime}\right) U_{n A}^{\mathrm{P}(\mathrm{E} 1)}(\mathbf{e}, \mathbf{k})}{E_{n}+E_{A^{\prime}}+\omega^{\prime}}\right|^{2} d \mathbf{n}_{\mathbf{k}} \mathbf{n}_{\mathbf{k}^{\prime}} d \omega^{\prime},
\end{array}
$$

the notations $U_{A^{\prime} n}^{\mathrm{P}(\mathrm{E} 1)}$ and $U_{A^{\prime} n}^{\mathrm{P}(\mathrm{E} 2)}$ for the dipole and quadrupole photons are used in accordance with decomposition Eq. (89). The Hamiltonian $\hat{H}$ in Eq. (89) acts on the eigenfunctions and, therefore, we can rewrite the expression Eq. (90) in the form

$$
\begin{array}{r}
d W_{A^{\prime} A}^{(\mathrm{E} 1 \mathrm{E} 2)}\left(\omega^{\prime}\right)=e^{4} \frac{\omega^{\prime 3} \omega^{3}}{4(2 \pi)^{3}} \sum_{m_{A} m_{A^{\prime}}} \frac{1}{2 j_{A}+1} \\
\times \mid \omega^{\prime} \sum_{n} \frac{\left(\mathbf{e}^{*} \mathbf{r}\right)_{A^{\prime} n}\left(\left(\mathbf{e}^{\prime *} \mathbf{r}\right)\left(\mathbf{n}_{\mathbf{k}^{\prime}} \mathbf{r}\right)\right)_{n A}}{E_{n}-E_{A}+\omega^{\prime}}+\omega \sum_{n} \frac{\left(\left(\mathbf{e}^{*} \mathbf{r}\right)\left(\mathbf{n}_{\mathbf{k}} \mathbf{r}\right)\right)_{A^{\prime} n}\left(\left(\mathbf{e}^{\prime *} \mathbf{r}\right)\right)_{n A}}{E_{n}-E_{A}+\omega^{\prime}} \\
+\omega \sum_{n} \frac{\left(\mathbf{e}^{\prime *} \mathbf{r}\right)_{A^{\prime} n}\left(\left(\mathbf{e}^{*} \mathbf{r}\right)\left(\mathbf{n}_{\mathbf{k}} \mathbf{r}\right)\right)_{n A}}{E_{n}-E_{A}+\omega}+\left.\omega^{\prime} \sum_{n} \frac{\left(\left(\mathbf{e}^{\prime *} \mathbf{r}\right)\left(\mathbf{n}_{\mathbf{k}^{\prime}} \mathbf{r}\right)\right)_{A^{\prime} n}\left(\left(\mathbf{e}^{*} \mathbf{r}\right)\right)_{n A}}{E_{n}-E_{A}+\omega}\right|^{2} d \mathbf{n}_{\mathbf{k}} \mathbf{n}_{\mathbf{k}^{\prime}} d \omega^{\prime},
\end{array}
$$

where the relation $\omega=E_{A}-E_{A^{\prime}}-\omega^{\prime}$ holds.

For the summation over polarizations we use again Eq. (13) and for integrating over the directions of the emitted photons we employ the formulas

$$
\begin{array}{r}
\int d \mathbf{n} n_{i}=\int d \mathbf{n} n_{i} n_{k} n_{l}=0, \int d \mathbf{n} n_{i} n_{k}=\frac{4 \pi}{3} \delta_{i k}, \\
\int d \mathbf{n} n_{i} n_{j} n_{k} n_{l}=\frac{4 \pi}{15}\left(\delta_{i j} \delta_{k l}+\delta_{i k} \delta_{j l}+\delta_{i l} \delta_{j k}\right) .
\end{array}
$$

The formula

$$
\sum_{\mathbf{e} \mathbf{e}^{\prime}}\left|\mathbf{e} \mathbf{e}^{\prime}\right|^{2}=1+\left(\mathbf{n}_{\mathbf{k}} \mathbf{n}_{\mathbf{k}^{\prime}}\right)^{2}=2-\left[\mathbf{n}_{\mathbf{k}} \times \mathbf{n}_{\mathbf{k}^{\prime}}\right]^{2}
$$

is also useful for calculations. We should stress that in our paper [15] an unfortunate misprint does exist: Eq. (51) should contain a vector product.

For the evaluation of the quadrupole matrix elements in Eq. (91) it is convenient to make use of the identity

$$
Y_{l_{1} m_{1}}(\mathbf{n}) Y_{l_{2} m_{2}}(\mathbf{n})=\sum_{L M} \sqrt{\frac{\left(2 l_{1}+1\right)\left(2 l_{2}+1\right)}{4 \pi(2 L+1)}} C_{l_{1} 0 l_{2} 0}^{L 0} C_{l_{1} m_{1} l_{2} m_{2}}^{L M} Y_{L M}(\mathbf{n}) .
$$

Therefore,

$$
\left(\mathbf{e}^{*} \mathbf{r}\right)\left(\mathbf{n}_{\mathbf{k}} \mathbf{r}\right)=\sqrt{\frac{2}{3}} \sqrt{\frac{4 \pi}{5}} \sum_{p_{1} p_{2} M}(-1)^{p_{1}+p_{2}} C_{2 \bar{M}}^{11}\left(p_{1} p_{2}\right) C_{1 p_{1} 1 p_{2}}^{2 \bar{M}} e_{p_{1}}^{*}\left(\mathbf{n}_{\mathbf{k}}\right)_{p_{2}} r^{2} Y_{2 M}\left(\mathbf{n}_{\mathbf{r}}\right)
$$


In this case the integration over angles can be provided by the (see [33]) standard relations

$$
\begin{gathered}
\int_{4 \pi} d \mathbf{n} Y_{l_{1} m_{1}}^{*}(\mathbf{n}) Y_{l_{2} m_{2}}(\mathbf{n})=\delta_{l_{1} l_{2}} \delta_{m_{1} m_{2}}, \\
\int_{4 \pi} d \mathbf{n} Y_{l_{1} m_{1}}^{*}(\mathbf{n}) Y_{l_{2} m_{2}}(\mathbf{n}) Y_{l_{3} m_{3}}(\mathbf{n})=\sqrt{\frac{\left(2 l_{2}+1\right)\left(2 l_{3}+1\right)}{4 \pi\left(2 l_{1}+1\right)}} C_{l_{2} 0 l_{3} 0}^{l_{1} 0} C_{l_{2} m_{2} l_{3} m_{3}}^{l_{1} m_{1}} .
\end{gathered}
$$

Finally, integrating Eq. 91) over angles and summing over all projections of the angular momenta, we receive

$$
d W_{2 \mathrm{p} 1 \mathrm{~s}}^{(\mathrm{E} 1 \mathrm{E} 2)}\left(\omega^{\prime}\right)=e^{4} \frac{\omega^{\prime 3} \omega}{(2 \pi)^{3} 5^{2} 3^{2}} \int_{4 \pi} d \mathbf{n}_{\mathbf{k}} \int_{4 \pi} d \mathbf{n}_{\mathbf{k}^{\prime}} \sum_{\mathbf{e e}^{\prime}}\left|\mathbf{e e}^{\prime}\right|^{2}\left[\omega^{\prime 2}\left[I_{1}\left(\omega^{\prime}\right)+I_{2}(\omega)\right]^{2}+\omega^{2}\left[I_{1}(\omega)+I_{2}\left(\omega^{\prime}\right)\right]^{2}\right] d \omega^{\prime}
$$

where $I_{1}(\omega), I_{2}(\omega)$ are defined by Eqs. 67, 68). After the summation over polarizations and integrating over photon directions the previous result Eqs. (66) and (69) are recovered.

The next step is the evaluation of E1M1 two-photon transition $2 \mathrm{p} \rightarrow 1 \mathrm{~s}+\gamma(\mathrm{M} 1)+\gamma(\mathrm{E} 1)$ with the set of quantum numbers of $\mathbf{e}$ and $\mathbf{k}$. Formula Eq. (90) can be cast into the form:

$$
\begin{array}{r}
d W_{A^{\prime} A}^{(\mathrm{E} 1 \mathrm{M} 1)}\left(\omega^{\prime}\right)=e^{4} \frac{\omega^{\prime}\left(E_{A}-E_{A^{\prime}}-\omega^{\prime}\right)}{(2 \pi)^{3}} \sum_{m_{A} m_{A^{\prime}}} \frac{1}{2 j_{A}+1} \\
\times \mid \sum_{n} \frac{U_{A^{\prime} n}^{\mathrm{P} 1)}(\mathbf{e}, \mathbf{k}) U_{n A}^{\mathrm{P}(\mathrm{M} 1)}\left(\mathbf{e}^{\prime}, \mathbf{k}^{\prime}\right)+U_{A^{\prime} n}^{\mathrm{P}(\mathrm{M} 1)}(\mathbf{e}, \mathbf{k}) U_{n A}^{\mathrm{P}(\mathrm{E} 1)}\left(\mathbf{e}^{\prime}, \mathbf{k}^{\prime}\right)}{E_{n}-E_{A}+\omega^{\prime}} \\
+\left.\sum_{n} \frac{U_{A^{\prime} n}^{\mathrm{P} 1)}\left(\mathbf{e}^{\prime}, \mathbf{k}^{\prime}\right) U_{n A}^{\mathrm{P}(\mathrm{M} 1)}(\mathbf{e}, \mathbf{k})+U_{A^{\prime} n}^{\mathrm{P} 1)}\left(\mathbf{e}^{\prime}, \mathbf{k}^{\prime}\right) U_{n A}^{\mathrm{P}(\mathrm{E} 1)}(\mathbf{e}, \mathbf{k})}{E_{n}+E_{A^{\prime}}+\omega^{\prime}}\right|^{2} d \mathbf{n}_{\mathbf{k}} \mathbf{n}_{\mathbf{k}^{\prime}} d \omega^{\prime}
\end{array}
$$

and, according to Eq. (89),

$$
\begin{aligned}
& d W_{A^{\prime} A}^{(\mathrm{E} 1 \mathrm{M} 1)}\left(\omega^{\prime}\right)=e^{4} \frac{\omega^{\prime} \omega}{4(2 \pi)^{3}} \frac{1}{2 j_{A}+1} \sum_{m_{A} m_{A^{\prime}}} \\
& \times \mid \omega \omega^{\prime} \sum_{n} \frac{\left(\mathbf{e}^{*} \mathbf{r}\right)_{A^{\prime} n}\left(\mathbf{e}^{\prime *}\left[\mathbf{n}_{\mathbf{k}^{\prime}} \times(\hat{\mathbf{l}}+2 \hat{\mathbf{s}})\right]\right)_{n A}}{E_{n}-E_{A}+\omega^{\prime}}+\omega \omega^{\prime} \frac{\left(\mathbf{e}^{*}\left[\mathbf{n}_{\mathbf{k}} \times(\hat{\mathbf{l}}+2 \hat{\mathbf{s}})\right)_{A^{\prime} n}\left(\mathbf{e}^{\prime *} \mathbf{r}\right)_{n A}\right.}{E_{n}-E_{A}+\omega^{\prime}} \\
& +\omega \omega^{\prime} \sum_{n} \frac{\left(\mathbf{e}^{* *} \mathbf{r}\right)_{A^{\prime} n}\left(\mathbf{e}^{*}\left[\mathbf{n}_{\mathbf{k}} \times(\hat{\mathbf{l}}+2 \hat{\mathbf{s}})\right]\right)_{n A}}{E_{n}-E_{A}+\omega}+\left.\omega \omega^{\prime} \frac{\left(\mathbf{e}^{* *}\left[\mathbf{n}_{\mathbf{k}^{\prime}} \times(\hat{\mathbf{l}}+2 \hat{\hat{s}})\right]\right)_{A^{\prime} n}\left(\mathbf{e}^{*} \mathbf{r}\right)_{n A}(\mathbf{e k})}{E_{n}-E_{A}+\omega}\right|^{2} d \mathbf{n}_{\mathbf{k}} \mathbf{n}_{\mathbf{k}^{\prime}} d \omega^{\prime},
\end{aligned}
$$

where $\hat{\mathbf{l}}=[\mathbf{r} \times \hat{\mathbf{p}}]$.

All the matrix elements can be easily evaluated with the aid of Eqs. (96)-(97). Finally, we arrive again at Eqs. (77)-(79):

$$
\begin{gathered}
d W_{2 \mathrm{p} 1 \mathrm{~s}}^{(\mathrm{E} 1 \mathrm{M} 1)}=e^{4} \frac{2^{5}}{\pi}\left(\frac{2}{3}\right)^{12}\left(3 / 8-\omega^{\prime}\right) \omega^{\prime}\left(\left(3 / 8-\omega^{\prime}\right)^{2}+\omega^{\prime 2}\right) d \omega^{\prime}, \\
W_{2 \mathrm{p} 1 \mathrm{~s}}^{\mathrm{E} 1 \mathrm{M} 1}=\frac{1}{2} \int_{0}^{3 / 8} d W_{2 p 1 s}^{\mathrm{E} 1 \mathrm{M} 1}=\frac{1}{\pi} \frac{1}{10935}(\alpha Z)^{8} \text { a.u. }=9.6769 \cdot 10^{-6} \mathrm{~s}^{-1}(Z=1) .
\end{gathered}
$$

Thus, in this section we have evaluated again the two-photon transition probabilites, E1E2 and E1M1, for the process of $2 p$ state decay in hydrogen atom. In contrast to [24], calculations were performed based on the representation dealing with the other set of quantum numbers. The analytic results obtained are in a good agreement with corresponding relativistic values (discrepancy is not more then $0.1 \%$ ). Recently the two-photon emission probabilities was evaluated by fully numerical methods in [44]. The corresponding results are in perfect agreement. The method applied in this section simplifies the determination of the dependence on directions of the emitted photons in the two-photon transition probability when the external field is present. 


\section{TWO-PHOTON DECAY OF THE 2S AND 2P HYDROGENIC STATES IN AN EXTERNAL ELECTRIC FIELD}

In this part of our work we evaluate the two-photon $2 \mathrm{~s}-1 \mathrm{~s}$ transition probability for the hydrogenic atoms in the presence of an external electric field. Similar as in section II of this paper we take into account only the mixing of the $2 \mathrm{~s}$ and $2 \mathrm{p}$ states. Such level mixing leads to additional E1E2 and E1M1 two-photon decays besides the dominant "pure" E1E1 two-photon transition. As it was shown in previous sections the E1E2 and E1M1 two-photon transition probabilities are about $(\alpha Z)^{2}$ times smaller then E1E1 transition probability. However, a consideration must be given to the fast increasing accuracy of the spectroscopical experiments. Therefore, E1E2 and E1M1 two-photon transition rates should be taken into account like a correction to E1E1 decay. Moreover, we will show that in the presence of the external electric field terms linear in the field will add to E1E1 transition probability; These are the interference terms. Furthermore, most if not all spectroscopical experiments (see most accurate experiments [4, 5]) involve an external electric field so the analysis of the two-photon decay transitions in an external electric field is indeed required.

In order to evaluate the two-photon $\overline{2 s} \rightarrow 1 \mathrm{~s}+2 \gamma$ transition probability in an external electric field we should turn to Eq. (88) and use the decomposition Eq. (89). The mixing of the $2 \mathrm{~s}$ and $2 p$ states is described by Eq. (16). According to Eq. (20), the two-photon transition probability in an external weak electric field can be introduced in the form:

$$
\begin{array}{r}
d W_{A^{\prime} A}^{(2 \gamma)}\left(\omega^{\prime}, \mathbf{n}_{\mathbf{k}}, \mathbf{n}_{\mathbf{k}^{\prime}}, \mathbf{e}, \mathbf{e}^{\prime}\right)=e^{4} \frac{\omega^{\prime} \omega}{(2 \pi)^{3}} \sum_{\mu^{\prime} \mu^{\prime \prime}} \frac{1}{2 j^{\prime \prime}+1} \mid \sum_{n} \frac{\left\langle 1 \mathrm{~s} \mu^{\prime}\left|A^{\mathrm{P}}(\mathbf{e}, \mathbf{k})\right| n\right\rangle\left\langle n\left|A^{\mathrm{P}}\left(\mathbf{e}^{\prime}, \mathbf{k}^{\prime}\right)\right| 2 \mathrm{~s} \mu^{\prime \prime}\right\rangle}{E_{n}-E_{A}+\omega^{\prime}} \\
-3 \eta \sum_{n} \sum_{\mu q}(-1)^{q} e D_{q} C_{1 \bar{q}, j^{\prime \prime} \mu^{\prime \prime}}^{j \mu} \frac{\left\langle 1 \mathrm{~s} \mu^{\prime}\left|A^{\mathrm{P}}(\mathbf{e}, \mathbf{k})\right| n\right\rangle\left\langle n\left|A^{\mathrm{P}}\left(\mathbf{e}^{\prime}, \mathbf{k}^{\prime}\right)\right| 2 \mathrm{p} \mu\right\rangle}{E_{n}-E_{A}+\omega^{\prime}}+ \\
+\sum_{n} \frac{\left\langle 1 \mathrm{~s} \mu^{\prime}\left|A^{\mathrm{P}}\left(\mathbf{e}^{\prime}, \mathbf{k}^{\prime}\right)\right| n\right\rangle\left\langle n\left|A^{\mathrm{P}}(\mathbf{e}, \mathbf{k})\right| 2 \mathrm{~s} \mu^{\prime \prime}\right\rangle}{E_{n}-E_{A}+\omega} \\
-\left.3 \eta \sum_{n} \sum_{\mu q}(-1)^{q} e D_{q} C_{1 \bar{q}, j^{\prime \prime} \mu^{\prime \prime}}^{j \mu} \frac{\left\langle 1 \mathrm{~s} \mu^{\prime}\left|A^{\mathrm{P}}\left(\mathbf{e}^{\prime}, \mathbf{k}^{\prime}\right)\right| n\right\rangle\left\langle n\left|A^{\mathrm{P}}(\mathbf{e}, \mathbf{k})\right| 2 \mathrm{p} \mu\right\rangle}{E_{n}-E_{A}+\omega}\right|^{2} d \mathbf{n}_{\mathbf{k}} d \mathbf{n}_{\mathbf{k}^{\prime}} d \omega^{\prime},
\end{array}
$$

where $A^{\mathrm{P}}(\mathbf{e}, \mathbf{k})$ is defined by Eq. 89$)$.

The decomposition Eq. (89) of the $A^{\mathrm{P}}(\mathbf{e}, \mathbf{k})$ operator shows that the first and third terms in Eq. (103) correspond to the E1E1 two-photon decay rate of the 2s electron level. Other terms in Eq. 103) represent the admixed E1E2 and E1M1 amplitudes of the 2p state two-photon decay probability. Application of the expansion Eq. (89) to the second term in Eq. (103) leads to the expression

$$
\begin{gathered}
\sum_{n} \frac{\left\langle 1 \mathrm{~s} \mu^{\prime}\left|A^{\mathrm{P}}(\mathbf{e}, \mathbf{k})\right| n\right\rangle\left\langle n\left|A^{\mathrm{P}}\left(\mathbf{e}^{\prime}, \mathbf{k}^{\prime}\right)\right| 2 \mathrm{p} \mu\right\rangle}{E_{n}-E_{A}+\omega^{\prime}}=-i \omega \omega^{\prime 2} \sum_{n} \frac{\left\langle 1 \mathrm{~s} \mu^{\prime}|\mathbf{e r}| n\right\rangle\left\langle n\left|\frac{1}{2}\left(\mathbf{e}^{\prime} \mathbf{r}\right)\left(\mathbf{n}_{\mathbf{k}^{\prime}} \mathbf{r}\right)\right| 2 \mathrm{p} \mu\right\rangle}{E_{n}-E_{A}+\omega^{\prime}} \\
+\omega \omega^{\prime} \sum_{n} \frac{\left\langle 1 \mathrm{~s} \mu^{\prime}\left|\mathbf{e}^{*} \mathbf{r}\right| n\right\rangle\left\langle n\left|\frac{1}{2}\left(\left[\mathbf{e}^{\prime} \times \mathbf{n}_{\mathbf{k}^{\prime}}\right](\hat{\mathbf{l}}+2 \hat{\mathbf{s}})\right)\right| 2 \mathrm{p} \mu\right\rangle}{E_{n}-E_{A}+\omega^{\prime}} \\
-i \omega^{\prime} \omega^{2} \sum_{n} \frac{\left\langle 1 \mathrm{~s} \mu^{\prime}\left|\frac{1}{2}(\mathbf{e r})\left(\mathbf{n}_{\mathbf{k}} \mathbf{r}\right)\right| n\right\rangle\left\langle n\left|\mathbf{e}^{\prime} \mathbf{r}\right| 2 \mathrm{p} \mu\right\rangle}{E_{n}-E_{A}+\omega^{\prime}} \\
+\omega \omega^{\prime} \sum_{n} \frac{\left\langle 1 \mathrm{~s} \mu^{\prime}\left|\frac{1}{2}\left(\left[\mathbf{e} \times \mathbf{n}_{\mathbf{k}}\right](\hat{\mathbf{l}}+2 \hat{\mathbf{s}})\right)\right| n\right\rangle\left\langle n\left|\mathbf{e}^{\prime *} \mathbf{r}^{\prime}\right| 2 \mathrm{p} \mu\right\rangle}{E_{n}-E_{A}+\omega^{\prime}} .
\end{gathered}
$$

In order to perform the summation over intermediate states in this expression we employ the Coulomb Green function method, which was described in the third section of this paper. We should note that in terms involving a magnetic dipole photon the Coulomb Green function does not occure due to the orthogonality of the radial functions. 
Summing over all angular momenta projections in the expression (103) we obtain

$$
\begin{gathered}
d W_{\overline{2 \mathrm{~s}} 1 \mathrm{~s}}^{(2 \gamma)}(\omega)=e^{4} \frac{\omega \omega^{\prime}}{2(2 \pi)^{3}}\left[\frac{2}{9} \omega^{2} \omega^{\prime 2}\left|\mathbf{e e}^{\prime}\right|^{2}\left[I_{1}^{2 \mathrm{~s} 1 \mathrm{~s}}(\omega)+I_{1}^{2 \mathrm{~s} 1 \mathrm{~s}}\left(\omega^{\prime}\right)\right]^{2}\right. \\
+\omega^{2} \omega^{\prime 2} \frac{2 \sqrt{3}}{3 \cdot 5} \frac{\Gamma_{2 \mathrm{p}}}{\Delta^{2}}\left|\mathbf{e e}^{\prime}\right|^{2}\left[I_{1}^{2 \mathrm{~s} 1 \mathrm{~s}}(\omega)+I_{1}^{2 \mathrm{~s} 1 \mathrm{~s}}\left(\omega^{\prime}\right)\right]\left[\omega^{\prime} I_{1}^{2 \mathrm{p} 1 \mathrm{~s}}\left(\omega^{\prime}\right)\left(e \mathbf{D} \mathbf{n}_{\mathbf{k}^{\prime}}\right)+\omega I_{1}^{2 \mathrm{p} 1 \mathrm{~s}}(\omega)\left(e \mathbf{D} \mathbf{n}_{\mathbf{k}}\right)\right] \\
+\omega \omega^{\prime} 2 \sqrt{2}\left(\frac{2}{3}\right)^{5} \frac{\Gamma_{2 \mathrm{p}}}{\Delta^{2}}\left[I_{1}^{2 \mathrm{~s} 1 \mathrm{~s}}(\omega)+I_{1}^{2 \mathrm{~s} 1 \mathrm{~s}}\left(\omega^{\prime}\right)\right]\left[\omega\left(\mathbf{e}\left[e \mathbf{D} \times\left[\mathbf{e}^{\prime} \times \mathbf{n}_{\mathbf{k}^{\prime}}\right]\right]\right)+\omega^{\prime}\left(\mathbf{e}^{\prime}\left[e \mathbf{D} \times\left[\mathbf{e} \times \mathbf{n}_{\mathbf{k}}\right]\right]\right)\right]\left(\mathbf{e e}^{\prime}\right)^{*} \\
+\omega^{2} \omega^{\prime 2} \frac{2 \sqrt{3}}{3 \cdot 5} \frac{\Gamma_{2 \mathrm{p}}}{\Delta^{2}}\left|\mathbf{e e}^{\prime}\right|^{2}\left[I_{1}^{2 \mathrm{~s} 1 \mathrm{~s}}(\omega)+I_{1}^{2 \mathrm{~s} 1 \mathrm{~s}}\left(\omega^{\prime}\right)\right]\left[\omega I_{2}^{2 \mathrm{p} 1 \mathrm{~s}}\left(\omega^{\prime}\right)\left(e \mathbf{D} \mathbf{n}_{\mathbf{k}}\right)+\omega^{\prime} I_{2}^{2 \mathrm{p} 1 \mathrm{~s}}(\omega)\left(e \mathbf{D} \mathbf{n}_{\mathbf{k}^{\prime}}\right)\right] \\
+\frac{2 \cdot 3}{5^{2} \Delta^{2}}\left[\left(\mathbf{e e}^{\prime}\right)\left(\mathbf{D n}_{\mathbf{k}^{\prime}}\right) \omega^{\prime} I_{1}^{2 \mathrm{p} 1 \mathrm{~s}}\left(\omega^{\prime}\right)+\left(\mathbf{e e}^{\prime}\right)\left(\mathbf{D n}_{\mathbf{k}}\right) \omega I_{1}^{2 \mathrm{p} 1 \mathrm{~s}}(\omega)\right]^{2} \\
+\frac{2 \cdot 3}{5^{2} \Delta^{2}}\left[\left(\mathbf{e e}^{\prime}\right)\left(\mathbf{D} \mathbf{n}_{\mathbf{k}}\right) \omega I_{2}^{2 \mathrm{p} 1 \mathrm{~s}}\left(\omega^{\prime}\right)+\left(\mathbf{e e}^{\prime}\right)\left(\mathbf{D n}_{\mathbf{k}^{\prime}}\right) \omega^{\prime} I_{2}^{2 \mathrm{p} 1 \mathrm{~s}}(\omega)\right]^{2} \\
+\frac{4 \cdot 3}{5^{2} \Delta^{2}}\left[\left(\mathbf{e e}^{\prime}\right)\left(\mathbf{D n}_{\mathbf{k}^{\prime}}\right) \omega^{\prime} I_{1}^{2 \mathrm{p} 1 \mathrm{~s}}\left(\omega^{\prime}\right)+\left(\mathbf{e} \mathbf{e}^{\prime}\right)\left(\mathbf{D} \mathbf{n}_{\mathbf{k}}\right) \omega I_{1}^{2 \mathrm{p} 1 \mathrm{~s}}(\omega)\right]\left[\left(\mathbf{e} \mathbf{e}^{\prime}\right)\left(\mathbf{D} \mathbf{n}_{\mathbf{k}}\right) \omega I_{2}^{2 \mathrm{p} 1 \mathrm{~s}}\left(\omega^{\prime}\right)+\left(\mathbf{e} \mathbf{e}^{\prime}\right)\left(\mathbf{D} \mathbf{n}_{\mathbf{k}^{\prime}}\right) \omega^{\prime} I_{2}^{2 \mathrm{p} 1 \mathrm{~s}}(\omega)\right] \\
+\frac{2^{14}}{3^{7} \Delta^{2}}\left[\omega\left([\mathbf{D} \times \mathbf{e}]\left[\mathbf{n}_{\mathbf{k}^{\prime}} \times \mathbf{e}^{\prime}\right]\right)+\omega^{\prime}\left(\left[\mathbf{D} \times \mathbf{e}^{\prime}\right]\left[\mathbf{n}_{\mathbf{k}} \times \mathbf{e}\right]\right)\right]^{2} \\
\left.+\frac{2^{15}}{3^{7} \Delta^{2}}\left[\omega(\mathbf{D e})\left[\mathbf{n}_{\mathbf{k}^{\prime}} \times \mathbf{e}^{\prime}\right]+\omega^{\prime}\left(\mathbf{D e}^{\prime}\right)\left[\mathbf{n}_{\mathbf{k}} \times \mathbf{e}\right]\right]^{2}\right] d \mathbf{n}_{\mathbf{k}} d \mathbf{n}_{\mathbf{k}^{\prime}} d \omega^{\prime}
\end{gathered}
$$

where $I_{1}^{2 \mathrm{~s} 1 \mathrm{~s}}(\omega)$ is defined by Eq. (60) and Eq. (61) for the 2s-1s transition, and $I_{1}^{2 \mathrm{p} 1 \mathrm{~s}}(\omega), I_{2}^{2 \mathrm{p} 1 \mathrm{~s}}(\omega)$ are defined by Eqs. (67), (68), correspondingly.

The summation over polarizations is provided by Eqs. (13) and (93). The radial integration can be analytically performed as well. Finally, the integration over $\omega^{\prime}$ is carried out with MATHEMATICA code. The result is

$$
\begin{gathered}
\frac{d W_{\overline{2 \mathrm{~s}} 1 \mathrm{~s}}^{(2 \gamma)}}{d \mathbf{n}_{\mathbf{k}} d \mathbf{n}_{\mathbf{k}^{\prime}}}=0.00131822(\alpha Z)^{6}-\frac{0.000230135}{\pi^{3}} \frac{e \Gamma_{2 \mathrm{p}}}{\Delta^{2}}\left[\mathbf{D n}_{\mathbf{k}}+\mathbf{D n}_{\mathbf{k}^{\prime}}\right]\left(1+\left(\mathbf{n}_{\mathbf{k}} \mathbf{n}_{\mathbf{k}^{\prime}}\right)^{2}\right)(\alpha Z)^{7} \\
\quad-\frac{0.0000340919}{\pi^{3}} \frac{e \Gamma_{2 \mathrm{p}}}{\Delta^{2}}\left[\mathbf{D n}_{\mathbf{k}}+\mathbf{D n}_{\mathbf{k}^{\prime}}\right]\left(1+\left(\mathbf{n}_{\mathbf{k}} \mathbf{n}_{\mathbf{k}^{\prime}}\right)\right)(\alpha Z)^{7}+0.00175091 \frac{e^{2} D^{2}}{\Delta^{2}}(\alpha Z)^{8} .
\end{gathered}
$$

The first term in this expression is the differential E1E1 2s-1s transition probability. The second and third terms represent interference terms for the mixed $2 \mathrm{~s}$ and $2 \mathrm{p}$ two-photon transition probabilities (second term corresponds to the E1E1.E1E2 transition probability and third one represents E1E1·E1M1 transition probability). Last term is related to the sum of the E1E2 and E1M1 two-photon probabilities, respectively.

Rewriting Eq. (106) in a form similar to Eq. (22) yields

$$
\frac{d W_{\overline{2 s} 1 \mathrm{~s}}^{(2 \gamma)}}{d \mathbf{n}_{\mathbf{k}} d \mathbf{n}_{\mathbf{k}^{\prime}}}=W_{0}\left[1 \pm \beta_{1}(D)\left[\mathbf{n}_{\mathbf{D}} \mathbf{n}_{\mathbf{k}}+\mathbf{n}_{\mathbf{D}} \mathbf{n}_{\mathbf{k}^{\prime}}\right]\left(1+\left(\mathbf{n}_{\mathbf{k}} \mathbf{n}_{\mathbf{k}^{\prime}}\right)^{2}\right) \pm \beta_{2}(D)\left[\mathbf{n}_{\mathbf{D}} \mathbf{n}_{\mathbf{k}}+\mathbf{n}_{\mathbf{D}} \mathbf{n}_{\mathbf{k}^{\prime}}\right]\left(1+\left(\mathbf{n}_{\mathbf{k}} \mathbf{n}_{\mathbf{k}^{\prime}}\right)\right)\right],
$$

where $W_{0}=W_{2 \mathrm{~s}}^{(2 \gamma)}+\widetilde{W}_{2 \mathrm{p}}^{(2 \gamma)} e^{2} D^{2} / \Delta^{2}, \widetilde{W}_{2 \mathrm{p}}^{(2 \gamma)}$ is the sum of the E1E2 and E1M1 transition probabilities.

Functions $\beta_{1}(D)$ and $\beta_{2}(D)$ are defined by

$$
\begin{gathered}
\beta_{1}(D)=\frac{0.000230135(\alpha Z)^{7}}{W_{0} \pi^{3}} \frac{|e| D \Gamma_{2 \mathrm{p}}}{\Delta^{2}}, \\
\beta_{2}(D)=\frac{0.0000340919(\alpha Z)^{7}}{W_{0} \pi^{3}} \frac{|e| D \Gamma_{2 \mathrm{p}}}{\Delta^{2}} .
\end{gathered}
$$

The maximum value for $\beta_{1}$ (or $\beta_{2}$ ) is achieved at the field strength

$$
D_{\max }=\frac{w^{2 \gamma} \Delta}{|e|} \approx \pm 0.000018 \text { a.u. } \approx \pm 57 \mathrm{kV} / \mathrm{cm},
$$

where the $(-)$ and $(+)$ signs in Eq. (110) correspond to the $\mathrm{H}$ and $\overline{\mathrm{H}}$ atoms, respectively. 
Then the corresponding maximum value of the transition rate $d W_{\overline{2} \mathrm{~s}}^{(2 \gamma)}$ is obtained via

$$
\begin{gathered}
\frac{d W_{\overline{2 s} 1 \mathrm{~s}}^{(2 \gamma)}}{d \mathbf{n}_{\mathbf{k}} d \mathbf{n}_{\mathbf{k}^{\prime}}}=W_{0}\left(D_{\max }\right) \\
\times\left[1 \pm 0.00024397\left[\mathbf{n}_{\mathbf{D}} \mathbf{n}_{\mathbf{k}}+\mathbf{n}_{\mathbf{D}} \mathbf{n}_{\mathbf{k}^{\prime}}\right]\left(1+\left(\mathbf{n}_{\mathbf{k}} \mathbf{n}_{\mathbf{k}^{\prime}}\right)^{2}\right) \pm 0.00003614\left[\mathbf{n}_{\mathbf{D}} \mathbf{n}_{\mathbf{k}}+\mathbf{n}_{\mathbf{D}} \mathbf{n}_{\mathbf{k}^{\prime}}\right]\left(1+\left(\mathbf{n}_{\mathbf{k}} \mathbf{n}_{\mathbf{k}^{\prime}}\right)\right)\right] .
\end{gathered}
$$

Integration over the directions of the emitted photons $\mathbf{n}_{\mathbf{k}}$ and $\mathbf{n}_{\mathbf{k}^{\prime}}$ leads to the value Eq. (62) for the E1E1 two-photon decay rate of the 2s-state, because the interference terms give zero result (see Eq. (92)). But for the differential transition probability Eq. (107) the interference terms clearly demonstrate the linear dependence on the external electric field $\mathbf{D}$. Thus the total two-photon $W_{\overline{2 s}}^{(2 \gamma)}$ transition probability integrated over photon directions is:

$$
W_{2 \mathrm{~s} 1 \mathrm{~s}}^{(2 \gamma)}\left(D_{\max }\right)=W_{0}\left(D_{\max }\right)=W_{2 \mathrm{~s}}^{(2 \gamma)}+\frac{\widetilde{W}_{2 \mathrm{p}}^{(2 \gamma)} e^{2} D_{\max }^{2}}{\Delta^{2}} \approx 3.98116 \cdot 10^{-16} \text { a.u. } \approx 16.4585 \mathrm{~s}^{-1},
$$

i.e. twice as large as the zero field value Eq. (58).

In principle, the dependece on the external electric field in the transition probability $W_{\overline{2 \mathrm{~s}}}^{(2 \gamma)}$ Eq. (111) can be considered as a correction which does not vanish after integration over the photons emission directions. If we return to the radiative correction considered in [12], then it is easy to see that the radiative correction (Eq. (36) in [12]) $\delta \Gamma_{2 s} / \Gamma_{2 s}=$ $2.020536 \frac{\alpha}{\pi}(\alpha Z)^{2} \ln \left[(\alpha Z)^{-2}\right]=-2.4594 \cdot 10^{-6}$ corresponds to the magnitude of the field $\left|D_{\mathrm{r}}\right| \approx D_{\max } \sqrt{\delta \Gamma_{2 \mathrm{~s}} / \Gamma_{2 \mathrm{~s}}} \approx$ $2.8 \cdot 10^{-8}$ a.u. $\approx 90 \mathrm{~V} / \mathrm{cm}$. Such fields are often used in the spectroscopic experiments, therefore, this effect also should be included in this context.

The linear over field corrections $\beta_{1}(D)$ and $\beta_{2}(D)$ in Eq. (107) reach the magnitude of the radiative correction at the fields approximately of the same order. Unlike the correction, discussed earlier in the section "2s decay rate for hydrogen and antihydrogen atoms in external electric fields", this is the correction directly to the same process, as radiative correction [12].

As it was mentioned above the formal T-noninvariance of the factor $\mathbf{n}_{\mathbf{D}} \mathbf{n}_{\mathbf{k}}$ in Eq. (21) and in Eq. 107) ( $\mathbf{n}_{\mathbf{k}}$ and $\mathbf{n}_{\mathbf{D}}$ are T-odd and T-even vectors, respectively) is compensated by the dependence on $\Gamma_{2 \mathrm{p}}$; This is the imitation of T-noninvariance in unstable systems, as predicted by Zeldovich [39].

The relative difference for the decay rates in $\mathrm{H}$ and $\overline{\mathrm{H}}$ atoms at the maximum value $D_{\max }$ equals to:

$$
\begin{gathered}
\frac{d W_{2 \mathrm{~s}}^{(2 \gamma)}(\mathrm{H})}{W_{0}\left(D_{\max }\right) d \mathbf{n}_{\mathbf{k}} d \mathbf{n}_{\mathbf{k}^{\prime}}}-\frac{d W_{2 \mathrm{~s}}^{(2 \gamma)}(\overline{\mathrm{H}})}{W_{0}\left(D_{\max }\right) d \mathbf{n}_{\mathbf{k}} d \mathbf{n}_{\mathbf{k}^{\prime}}}= \\
=2 \beta_{1}\left(D_{\max }\right)\left(\mathbf{n}_{\mathbf{D}} \mathbf{n}_{\mathbf{k}}+\mathbf{n}_{\mathbf{D}} \mathbf{n}_{\mathbf{k}^{\prime}}\right)\left(1+\left(\mathbf{n}_{\mathbf{k}} \mathbf{n}_{\mathbf{k}^{\prime}}\right)^{2}\right)+2 \beta_{2}\left(D_{\max }\right)\left(\mathbf{n}_{\mathbf{D}} \mathbf{n}_{\mathbf{k}}+\mathbf{n}_{\mathbf{D}} \mathbf{n}_{\mathbf{k}^{\prime}}\right)\left(1+\mathbf{n}_{\mathbf{k}} \mathbf{n}_{\mathbf{k}^{\prime}}\right) \\
=\left(\mathbf{n}_{\mathbf{D}} \mathbf{n}_{\mathbf{k}}+\mathbf{n}_{\mathbf{D}} \mathbf{n}_{\mathbf{k}^{\prime}}\right)\left(0.000280111+0.00024397\left(\mathbf{n}_{\mathbf{k}} \mathbf{n}_{\mathbf{k}^{\prime}}\right)+0.0000361414 \mathbf{n}_{\mathbf{k}} \mathbf{n}_{\mathbf{k}^{\prime}}\right) .
\end{gathered}
$$

This ratio is close to $0.028 \%$ and represents a tiny effect reflecting the difference between matter and anti-matter even at maximum field strength $D_{\max }$.

For completeness the $\overline{2 p} \rightarrow 1 \mathrm{~s}+2 \gamma$ two-photon transition probability should be considered as well. It can be evaluated similarly to $\overline{2 \mathrm{~s}} \rightarrow 1 \mathrm{~s}+2 \gamma$ two-photon decay rate with the use of the wave function

$$
\left|\overline{2 \mathrm{p}} \mu^{\prime \prime}\right\rangle=\left|2 \mathrm{p} \mu^{\prime \prime}\right\rangle-\eta \sum_{\mu}\left\langle 2 \mathrm{~s} \mu^{\prime \prime}|e \mathbf{D r}| 2 \mathrm{p} \mu\right\rangle\left|2 \mathrm{~s} \mu^{\prime \prime}\right\rangle .
$$

In this case the two-photon transition without an external electric field will be provided by the sum of the E1E2 and E1M1 decays, and the interference terms will be the same as in Eq. (105).

The result can be presented in the form

$$
\begin{array}{r}
d W_{\overline{2 \mathrm{p}} 1 \mathrm{~s}}^{(2 \gamma)}=\left[d W_{2 \mathrm{p} 1 \mathrm{~s}}^{\mathrm{E} 1 \mathrm{E} 2}+d W_{2 \mathrm{p} 1 \mathrm{~s}}^{\mathrm{E} 1 \mathrm{M} 1}+\frac{9 e^{2} D^{2}}{\Delta^{2}} d W_{2 \mathrm{~s} 1 \mathrm{~s}}^{\mathrm{E} 1 \mathrm{E} 1}+\frac{0.000230135}{\pi^{3}} \frac{\Gamma_{2 \mathrm{p}}}{\Delta^{2}}\left[e \mathbf{D n}_{\mathbf{k}}+e \mathbf{D n}_{\mathbf{k}^{\prime}}\right]\left(1+\left(\mathbf{n}_{\mathbf{k}} \mathbf{n}_{\mathbf{k}^{\prime}}\right)^{2}\right)(\alpha Z)^{7}\right. \\
\left.+\frac{0.0000340919}{\pi^{3}} \frac{\Gamma_{2 \mathrm{p}}}{\Delta^{2}}\left[e \mathbf{D n}_{\mathbf{k}}+e \mathbf{D n}_{\mathbf{k}^{\prime}}\right]\left(1+\mathbf{n}_{\mathbf{k}} \mathbf{n}_{\mathbf{k}^{\prime}}\right)(\alpha Z)^{7}\right] d \mathbf{n}_{\mathbf{k}} d \mathbf{n}_{\mathbf{k}^{\prime}}
\end{array}
$$

$$
\frac{d W_{\frac{2 \gamma}{2 p} 1 \mathrm{~s}}^{(2 \gamma)}}{d \mathbf{n}_{\mathbf{k}} d \mathbf{n}_{\mathbf{k}^{\prime}}}=W_{0}\left[1 \mp \beta_{1}(D)\left[\mathbf{n}_{\mathbf{D}} \mathbf{n}_{\mathbf{k}}+\mathbf{n}_{\mathbf{D}} \mathbf{n}_{\mathbf{k}^{\prime}}\right]\left(1+\left(\mathbf{n}_{\mathbf{k}} \mathbf{n}_{\mathbf{k}^{\prime}}\right)^{2}\right) \mp \beta_{2}(D)\left[\mathbf{n}_{\mathbf{D}} \mathbf{n}_{\mathbf{k}}+\mathbf{n}_{\mathbf{D}} \mathbf{n}_{\mathbf{k}^{\prime}}\right]\left(1+\mathbf{n}_{\mathbf{k}} \mathbf{n}_{\mathbf{k}^{\prime}}\right)\right],
$$

where $W_{0}=W_{2 \mathrm{p} 1 \mathrm{~s}}^{(\mathrm{E} 1 \mathrm{E} 2)}+W_{2 \mathrm{p} 1 \mathrm{~s}}^{(\mathrm{E} 1 \mathrm{M} 1)}+9 e^{2} D^{2} W_{2 \mathrm{~s} 1 \mathrm{~s}}^{(\mathrm{E} 1 \mathrm{E})} / \Delta^{2}$ and the functions $\beta_{1}(D), \beta_{2}(D)$ are defined again by Eq. (108). 
Then the maximum of the $\beta_{1}$ (or $\beta_{2}$ ) is achieved at

$$
\left|D_{\max }\right|=\frac{\Delta}{3|e| w^{2 \gamma}} \approx 7.1 \cdot 10^{-11} \text { a.u. } \approx 0.23 \mathrm{~V} / \mathrm{cm}
$$

The corresponding maximum value of $d W_{\overline{2 p}}^{(2 \gamma)}$ is

$$
\begin{gathered}
\frac{d W_{\frac{2 \gamma}{2 \gamma}}^{(2 \gamma}}{d \mathbf{n}_{\mathbf{k}} d \mathbf{n}_{\mathbf{k}^{\prime}}}=W_{0}\left(D_{\max }\right) \\
\times\left[1 \mp 0.00048613\left[\mathbf{n}_{\mathbf{D}} \mathbf{n}_{\mathbf{k}}+\mathbf{n}_{\mathbf{D}} \mathbf{n}_{\mathbf{k}^{\prime}}\right]\left(1+\left(\mathbf{n}_{\mathbf{k}} \mathbf{n}_{\mathbf{k}^{\prime}}\right)^{2}\right) \mp 0.000720147\left[\mathbf{n}_{\mathbf{D}} \mathbf{n}_{\mathbf{k}}+\mathbf{n}_{\mathbf{D}} \mathbf{n}_{\mathbf{k}^{\prime}}\right]\left(1+\mathbf{n}_{\mathbf{k}} \mathbf{n}_{\mathbf{k}^{\prime}}\right)\right] .
\end{gathered}
$$

After integration over $\mathbf{n}_{\mathbf{k}}$ and $\mathbf{n}_{\mathbf{k}^{\prime}}$ in Eq. (114) the term quadratic in the external electric field for the correction to the two-photon transition probability $2 \mathrm{p}-1 \mathrm{~s}$ still remains:

$$
W_{\overline{2 \mathrm{p}} 1 \mathrm{~s}}^{(2 \gamma)}\left(D_{\max }\right)=W_{2 \mathrm{p} 1 \mathrm{~s}}^{(\mathrm{E} 1 \mathrm{E} 2)}+W_{2 \mathrm{p} 1 \mathrm{~s}}^{(\mathrm{E} 1 \mathrm{M} 1)}+\frac{9 e^{2} D_{\max }^{2} W_{2 \mathrm{~s} 1 \mathrm{~s}}^{(\mathrm{E} 1 \mathrm{E} 1)}}{\Delta^{2}} \approx 4.09 \cdot 10^{-22} \mathrm{a} . \mathrm{u} . \approx 1.69 \cdot 10^{-5} \mathrm{~s}^{-1} .
$$

Finally, the $(+)$ and $(-)$ signs in Eq. (117) correspond to the $\mathrm{H}$ and $\overline{\mathrm{H}}$ atoms, respectively. The relative difference for the decay rates in $\mathrm{H}$ and $\overline{\mathrm{H}}$ atoms at the maximum value $D_{\max }$ equals to:

$$
\begin{gathered}
\frac{d W \frac{2 \gamma}{2 \mathrm{p}} 1 \mathrm{~s}}{W_{0}\left(D_{\max }\right) d \mathbf{n}_{\mathbf{k}} d \mathbf{n}_{\mathbf{k}^{\prime}}}(\mathrm{H})-\frac{d W_{\overline{\mathrm{p}}} 1 \mathrm{~s}}{W_{0}\left(D_{\max }\right) d \mathbf{n}_{\mathbf{k}} d \mathbf{n}_{\mathbf{k}^{\prime}}}(\overline{\mathrm{H}})= \\
=2 \beta_{1}\left(D_{\max }\right)\left(\mathbf{n}_{\mathbf{D}} \mathbf{n}_{\mathbf{k}}+\mathbf{n}_{\mathbf{D}} \mathbf{n}_{\mathbf{k}^{\prime}}\right)\left(1+\left(\mathbf{n}_{\mathbf{k}} \mathbf{n}_{\mathbf{k}^{\prime}}\right)^{2}\right)+2 \beta_{2}\left(D_{\max }\right)\left(\mathbf{n}_{\mathbf{D}} \mathbf{n}_{\mathbf{k}}+\mathbf{n}_{\mathbf{D}} \mathbf{n}_{\mathbf{k}^{\prime}}\right)\left(1+\mathbf{n}_{\mathbf{k}} \mathbf{n}_{\mathbf{k}^{\prime}}\right) \\
\left.=\left(\mathbf{n}_{\mathbf{D}} \mathbf{n}_{\mathbf{k}}\right)+\mathbf{n}_{\mathbf{D}} \mathbf{n}_{\mathbf{k}^{\prime}}\right)\left(0.0111629+0.0097226\left(\mathbf{n}_{\mathbf{k}} \mathbf{n}_{\mathbf{k}^{\prime}}\right)^{2}+0.00144029 \mathbf{n}_{\mathbf{k}} \mathbf{n}_{\mathbf{k}^{\prime}}\right) .
\end{gathered}
$$

This ratio turns out to be close to $1 \%$. However, any direct observation of this difference should be difficult due to the huge background from the one-photon transition $2 \mathrm{p} \rightarrow 1 \mathrm{~s}+\gamma$.

\section{THREE-PHOTON DECAY RATE FOR THE $2 p$ STATE OF HYDROGEN-LIKE LIGHT ATOMIC SYSTEMS.}

In this last section we present the calculation of the E1E1E1 transition probability for the 2p hydrogenic state decay. Parametric estimate can be easily obtained in usual way and it is $\alpha(\alpha Z)^{8}$ a.u. Therefore, one can expect a numerical result for the E1E1E1 decay rate which is comparable with values obtained for the E1E2 and E1M1 transition.

According to the Feynman rules the $S$-matrix element for the $n$-photons emission process with the transition from the state $A$ to $A^{\prime}\left(A \rightarrow n \gamma+A^{\prime}\right)$ can be written as (in r.u.):

$$
S_{A^{\prime} A}^{(n)}=(-i e)^{n} \int\left(\bar{\psi}_{A^{\prime}}\left(x_{1}\right) \hat{A}\left(x_{1}\right) S\left(x_{1}, x_{2}\right) \hat{A}\left(x_{2}\right) \ldots \hat{A}\left(x_{n-1}\right) S\left(x_{n-1}, x_{n}\right) \psi_{A}\left(x_{n}\right)\right) d x_{1} \ldots d x_{n}
$$

where $\hat{A}$ is the emission operator, $\psi_{A}, \bar{\psi}_{A^{\prime}}$ are Dirac wave function, $S\left(x_{n-1}, x_{n}\right)$ etc. denotes the bound electron propagator, and $e$ is the electron charge.

The emission operator is given by

$$
\hat{A}(x)=\sqrt{\frac{2 \pi}{\omega}} \hat{e}^{(\lambda)} e^{-i(\mathbf{k r}-\omega t)}
$$

where $\hat{e}^{(\lambda)}$ is the 4-vector of the photon polarization $\left(\hat{e}^{(\lambda)}=\mathbf{e} \gamma\right.$, e is the polarization vector, $\gamma$ are the Dirac matrices) and $\mathbf{k}$ is the wave vector. In what follows we employ the nonrelativistic approximation, replacing the Dirac solutions for atomic electron orbitals by the corresponding Schrödinger ones and omitting, where it is justified, the contributions of the negative energy states to the exact electron propagators in Eq. 120).

Integrating over time variables in Eq. (120) results in

$$
S_{A^{\prime} A}^{(n)}=-2 \pi i(-i e)^{n}\left(\frac{2 \pi}{\omega}\right)^{n / 2} \delta\left(E_{A^{\prime}}+n \omega-E_{A}\right) \sum_{s_{1}, \ldots, s_{n-1}} \frac{\left(\hat{e} e^{i \mathbf{k r}}\right)_{A^{\prime} s_{n-1}} \ldots\left(\hat{e} e^{i \mathbf{k r}}\right)_{s_{1} A}}{\left[E_{s_{n-1}}-E_{A}-(n-1) \omega\right] \ldots\left[E_{s_{1}}-E_{A}-\omega\right]},
$$


where the summation over $s_{i}$ extends over all intermediate Schrödinger states with positive energy, $E_{s}$ are the Schrödinger energies for an atomic electron.

In the nonrelativistic approximation we can expand the exponents $e^{i \mathbf{k r}}$ in Eq. (122), leaving only the first term and replace the matrix elements $(\hat{e})_{s s^{\prime}}$ with Dirac wave functions by the matrix elements $(\mathbf{e} \hat{\mathbf{p}})_{s s^{\prime}}$, with Schrödinger wave functions, where $\hat{\hat{\mathbf{p}}}$ is the electron momentum operator. Then, using a well known quantum mechanical relation $\left([\mathbf{r}, \hat{H}]_{-}\right)_{s s^{\prime}}=i(\hat{\mathbf{p}})_{s s^{\prime}}$, the amplitude of the three-photon emission process can be written in a form:

$$
\begin{array}{r}
U_{A^{\prime} A}^{(3)}=-(-i e)^{3}(2 \pi)^{3 / 2} \sqrt{\omega_{1} \omega_{2} \omega_{3}} \delta\left(E_{A^{\prime}}+\omega_{1}+\omega_{2}+\omega_{3}-E_{A}\right) \times \\
{\left[\sum_{s_{1}, s_{2}} \frac{\left(\mathbf{e}_{1}^{*} \mathbf{r}_{1}\right)_{A^{\prime} s_{2}}\left(\mathbf{e}_{2}^{*} \mathbf{r}_{2}\right)_{s_{2} s_{1}}\left(\mathbf{e}_{3}^{*} \mathbf{r}_{3}\right)_{s_{1} A}}{\left(E_{s_{2}}-E_{A}+\omega_{1}+\omega_{2}\right)\left(E_{s_{1}}-E_{A}+\omega_{3}\right)}+\sum_{s_{1}, s_{2}} \frac{\left(\mathbf{e}_{1}^{*} \mathbf{r}_{1}\right)_{A^{\prime} s_{2}}\left(\mathbf{e}_{3}^{*} \mathbf{r}_{3}\right)_{s_{2} s_{1}}\left(\mathbf{e}_{2}^{*} \mathbf{r}_{2}\right)_{s_{1} A}}{\left(E_{s_{2}}-E_{A}+\omega_{1}+\omega_{3}\right)\left(E_{s_{1}}-E_{A}+\omega_{2}\right)}\right.} \\
+\sum_{s_{1}, s_{2}} \frac{\left(\mathbf{e}_{2}^{*} \mathbf{r}_{2}\right)_{A^{\prime} s_{2}}\left(\mathbf{e}_{1}^{*} \mathbf{r}_{1}\right)_{s_{2} s_{1}}\left(\mathbf{e}_{3}^{*} \mathbf{r}_{3}\right)_{s_{1} A}}{\left(E_{s_{2}}-E_{A}+\omega_{1}+\omega_{2}\right)\left(E_{s_{1}}-E_{A}+\omega_{3}\right)}+\sum_{s_{1}, s_{2}} \frac{\left(\mathbf{e}_{2}^{*} \mathbf{r}_{2}\right)_{A^{\prime} s_{2}}\left(\mathbf{e}_{3}^{*} \mathbf{r}_{3}\right)_{s_{2} s_{1}}\left(\mathbf{e}_{1}^{*} \mathbf{r}_{1}\right)_{s_{1} A}}{\left(E_{s_{2}}-E_{A}+\omega_{2}+\omega_{3}\right)\left(E_{s_{1}}-E_{A}+\omega_{1}\right)} \\
\left.+\sum_{s_{1}, s_{2}} \frac{\left(\mathbf{e}_{3}^{*} \mathbf{r}_{3}\right)_{A^{\prime} s_{2}}\left(\mathbf{e}_{1}^{*} \mathbf{r}_{1}\right)_{s_{2} s_{1}}\left(\mathbf{e}_{2}^{*} \mathbf{r}_{2}\right)_{s_{1} A}}{\left(E_{s_{2}}-E_{A}+\omega_{1}+\omega_{3}\right)\left(E_{s_{1}}-E_{A}+\omega_{2}\right)}+\sum_{s_{1}, s_{2}} \frac{\left(\mathbf{e}_{3}^{*} \mathbf{r}_{3}\right)_{A^{\prime} s_{2}}\left(\mathbf{e}_{2}^{*} \mathbf{r}_{2}\right)_{s_{2} s_{1}}\left(\mathbf{e}_{1}^{*} \mathbf{r}_{1}\right)_{s_{1} A}}{\left(E_{s_{2}}-E_{A}+\omega_{2}+\omega_{3}\right)\left(E_{s_{1}}-E_{A}+\omega_{1}\right)}\right] .
\end{array}
$$

Here $\omega_{1}, \omega_{2}, \omega_{3}$ are the frequencies (energies) of the emitted photon.

The probability of the three-photon emission process is

$$
d W_{A^{\prime} A}^{(3)}=2 \pi\left|U_{A^{\prime} A}^{(3)}\right|^{2} \delta\left(E_{A^{\prime}}+\omega_{1}+\omega_{2}+\omega_{3}-E_{A}\right) \frac{d \mathbf{k}_{\mathbf{1}} d \mathbf{k}_{\mathbf{2}} d \mathbf{k}_{\mathbf{3}}}{(2 \pi)^{9}} .
$$

The total probability can be received from Eq. (124) by the summation over photon polarizations $\mathbf{e}_{1}, \mathbf{e}_{2}, \mathbf{e}_{3}$ and integration over all the photon-emission directions $\mathbf{k}_{1}, \mathbf{k}_{2}, \mathbf{k}_{3}$ and frequencies $\omega_{1}, \omega_{2}, \omega_{3}$. For the summation over polarizations and integration over photon directions it is convenient to use the relations Eqs. (13), (92), (93).

After averaging over angular momentum projections of the initial state and summing over final ones, the probability of the three-photon emission process results as

$$
\begin{array}{r}
d W_{A A^{\prime}}^{(3)}=e^{9} 2^{4} \omega_{1}^{3} \omega_{2}^{3} \omega_{3}^{3} \frac{d \omega_{1} d \omega_{2} d \omega_{3}}{\pi^{2}} \delta\left(E_{A^{\prime}}+\omega_{1}+\omega_{2}+\omega_{3}-E_{A}\right) \times \\
\sum_{m_{l_{A^{\prime}}} m_{l_{A}}} \frac{1}{2 l_{A}+1} \sum_{q_{1} q_{2} q_{3}}(-1)^{q_{1}+q_{2}+q_{3}} U_{A^{\prime} A}^{(3)}\left(q_{1}, q_{2}, q_{3}\right) U_{A^{\prime} A}^{(3) *}\left(-q_{1},-q_{2},-q_{3}\right),
\end{array}
$$

where the expression for the amplitude $U_{A^{\prime} A}^{(3)}\left(q_{1}, q_{2}, q_{3}\right)$ in spherical representation is given by

$$
\begin{aligned}
U_{A^{\prime} A}^{(3)}\left(q_{1}, q_{2}, q_{3}\right)= & {\left[\sum_{s_{1} s_{2}} \frac{\left(\left(\mathbf{r}_{1}\right)_{q_{1}}\right)_{A^{\prime} s_{2}}\left(\left(\mathbf{r}_{2}\right)_{q_{2}}\right)_{s_{2} s_{1}}\left(\left(\mathbf{r}_{3}\right)_{q_{3}}\right)_{s_{1} A}}{\left(E_{s_{2}}-E_{A}+\omega_{1}+\omega_{2}\right)\left(E_{s_{1}}-E_{A}+\omega_{3}\right)}+\sum_{s_{1} s_{2}} \frac{\left(\left(\mathbf{r}_{1}\right)_{q_{1}}\right)_{A^{\prime} s_{2}}\left(\left(\mathbf{r}_{3}\right)_{q_{3}}\right)_{s_{2} s_{1}}\left(\left(\mathbf{r}_{2}\right)_{q_{2}}\right)_{s_{1} A}}{\left(E_{s_{2}}-E_{A}+\omega_{1}+\omega_{3}\right)\left(E_{s_{1}}-E_{A}+\omega_{2}\right)}\right.} \\
& +\sum_{s_{1} s_{2}} \frac{\left(\left(\mathbf{r}_{2}\right)_{q_{2}}\right)_{A^{\prime} s_{2}}\left(\left(\mathbf{r}_{1}\right)_{q_{1}}\right)_{s_{2} s_{1}}\left(\left(\mathbf{r}_{3}\right)_{q_{3}}\right)_{s_{1} A}}{\left(E_{s_{2}}-E_{A}+\omega_{1}+\omega_{2}\right)\left(E_{s_{1}}-E_{A}+\omega_{3}\right)}+\sum_{s_{1} s_{2}} \frac{\left(\left(\mathbf{r}_{2}\right)_{q_{2}}\right)_{A^{\prime} s_{2}}\left(\left(\mathbf{r}_{3}\right)_{q_{3}}\right)_{s_{2} s_{1}}\left(\left(\mathbf{r}_{1}\right)_{q_{1}}\right)_{s_{1} A}}{\left(E_{s_{2}}-E_{A}+\omega_{2}+\omega_{3}\right)\left(E_{s_{1}}-E_{A}+\omega_{1}\right)} \\
& \left.+\sum_{s_{1} s_{2}} \frac{\left(\left(\mathbf{r}_{3}\right)_{q_{3}}\right)_{A^{\prime} s_{2}}\left(\left(\mathbf{r}_{1}\right)_{q_{1}}\right)_{s_{2} s_{1}}\left(\left(\mathbf{r}_{2}\right)_{q_{2}}\right)_{s_{1} A}}{\left(E_{s_{2}}-E_{A}+\omega_{1}+\omega_{3}\right)\left(E_{s_{1}}-E_{A}+\omega_{2}\right)}+\sum_{s_{1} s_{2}} \frac{\left(\left(\mathbf{r}_{3}\right)_{q_{3}}\right)_{A^{\prime} s_{2}}\left(\left(\mathbf{r}_{2}\right)_{q_{2}}\right)_{s_{2} s_{1}}\left(\left(\mathbf{r}_{1}\right)_{q_{1}}\right)_{s_{1} A}}{\left(E_{s_{2}}-E_{A}+\omega_{2}+\omega_{3}\right)\left(E_{s_{1}}-E_{A}+\omega_{1}\right)}\right] .
\end{aligned}
$$

In order to calculate the transition probabilities for the process $2 \mathrm{p} \rightarrow 3 \gamma(\mathrm{E} 1)+1$ s in the hydrogen atom the nonrelativistic Coulomb Green function method is employed. Inserting the Green function in a form Eq. (50) in (126) and representing the vector component $(\mathbf{r})_{q}$ like $r_{q}=\sqrt{\frac{4 \pi}{3}} Y_{1 q}$ we can perform the angular integration, which gives

$$
\begin{aligned}
& U_{A^{\prime} A}^{(3)}\left(q_{1}, q_{2}, q_{3}\right)=\sqrt{\frac{2 l_{A}+1}{2 l_{A^{\prime}}+1}} \sum_{l_{1} m_{l_{1}}} \sum_{l_{2} m_{l_{2}}} C_{10 l_{2} 0}^{l_{A^{\prime}} 0} C_{10 l_{1} 0}^{l_{2} 0} C_{10 l_{A} 0}^{l_{1} 0}\left\{F _ { l _ { 1 } l _ { 2 } } ( \nu _ { 1 } , \nu _ { 2 } ) \left[C_{1 q_{1} l_{2} m_{l_{2}}}^{l_{A^{\prime}} m_{A^{\prime}}} C_{1 q_{2} l_{1} m_{l_{1}}}^{l_{2} m_{l_{2}}} C_{1 q_{3} l_{A} m_{A}}^{l_{1} m_{l_{1}}}\right.\right. \\
& \left.C_{1 q_{2} l_{2} m_{l_{2}}}^{l_{A^{\prime}} m_{A^{\prime}}} C_{1 q_{1} l_{1} m_{l_{1}}}^{l_{2} m_{l_{2}}} C_{1 q_{3} l_{A} m_{A}}^{l_{1} m_{l_{1}}}\right]+F_{l_{1} l_{2}}\left(\nu_{3}, \nu_{4}\right)\left[C_{1 q_{1} l_{2} m_{l_{2}}}^{l_{A^{\prime}} m_{A^{\prime}}} C_{1 q_{3} l_{1} m_{l_{1}}}^{l_{2} m_{l_{2}}} C_{1 q_{2} l_{A} m_{A}}^{l_{1} m_{l_{1}}}+C_{1 q_{3} l_{2} m_{l_{2}}}^{l_{A^{\prime}} m_{A^{\prime}}} C_{1 q_{1} l_{1} m_{l_{1}}}^{l_{2} m_{l_{2}}} C_{1 q_{2} l_{A} m_{A}}^{l_{1} m_{l_{1}}}\right] \\
& \left.+F_{l_{1} l_{2}}\left(\nu_{5}, \nu_{6}\right)\left[C_{1 q_{2} l_{2} m_{l_{2}}}^{l_{A^{\prime}} m_{A^{\prime}}} C_{1 q_{3} l_{1} m_{l_{1}}}^{l_{2} m_{l_{2}}} C_{1 q_{1} l_{A} m_{A}}^{l_{1} m_{l_{1}}}+C_{1 q_{3} l_{2} m_{l_{2}}}^{l_{A^{\prime}} m_{A^{\prime}}} C_{1 q_{2} l_{1} m_{l_{1}}}^{l_{2} m_{l_{2}}} C_{1 q_{1} l_{A} m_{A}}^{l_{1} m_{l_{1}}}\right]\right\} \text {. }
\end{aligned}
$$


Here $C_{l_{1} m_{l_{1}} l_{2} m_{l_{2}}}^{l_{3} m_{l_{3}}}$ is the Clebsch-Gordan coefficient, and

$$
\begin{gathered}
F_{l_{1}, l_{2}}\left(\nu_{i}, \nu_{j}\right)=\int_{0}^{\infty} \int_{0}^{\infty} \int_{0}^{\infty} d r_{1} d r_{2} d r_{3} r_{1}^{3} r_{2}^{3} r_{3}^{3} R_{n_{A^{\prime}} l_{A^{\prime}}}\left(r_{1}\right) g_{l_{2}}\left(\nu_{i} ; r_{1}, r_{2}\right) g_{l_{1}}\left(\nu_{j} ; r_{2}, r_{3}\right) R_{n_{A}, l_{A}}\left(r_{3}\right), \\
\nu_{1}=Z / \sqrt{-2\left(E_{A}-\omega_{1}-\omega_{2}\right)}, \nu_{2}=Z / \sqrt{-2\left(E_{A}-\omega_{3}\right)}, \nu_{3}=Z / \sqrt{-2\left(E_{A}-\omega_{1}-\omega_{3}\right)} \\
\nu_{4}=Z / \sqrt{-2\left(E_{A}-\omega_{2}\right)}, \nu_{5}=Z / \sqrt{-2\left(E_{A}-\omega_{2}-\omega_{3}\right)}, \nu_{6}=Z / \sqrt{-2\left(E_{A}-\omega_{1}\right)}
\end{gathered}
$$

corresponding to the 6 different terms in Eq. (126).

For the $2 \mathrm{p} \rightarrow 3 \gamma(\mathrm{E} 1)+1$ s process, $R_{n_{A}, l_{A}} \equiv R_{2 \mathrm{p}}, R_{n_{A^{\prime}} l_{A^{\prime}}} \equiv R_{1 \mathrm{~s}}, l_{A}=1, l_{A^{\prime}}=0$. Performing the summation over all angular momentum projections in Eq. 125) we arrive at the expression

$$
\begin{array}{r}
d W_{2 \mathrm{p} 1 \mathrm{~s}}^{(3)}=e^{9} \omega_{1}^{3} \omega_{2}^{3} \omega_{3}^{3} \frac{d \omega_{1} d \omega_{2} d \omega_{3}}{5 \cdot 3^{3} \pi^{2}} \frac{2^{6}}{3} \delta\left(E_{1 \mathrm{~s}}+\omega_{1}+\omega_{2}+\omega_{3}-E_{2 \mathrm{p}}\right)\left[15 F_{01}\left(\nu_{1}, \nu_{2}\right)^{2}+\right. \\
+15 F_{01}\left(\nu_{3}, \nu_{4}\right)^{2}+15 F_{01}\left(\nu_{5}, \nu_{6}\right)^{2}+20 F_{01}\left(\nu_{5}, \nu_{6}\right) F_{21}\left(\nu_{1}, \nu_{2}\right)+12 F_{21}\left(\nu_{1}, \nu_{2}\right)^{2}+20 F_{01}\left(\nu_{5}, \nu_{6}\right) F_{21}\left(\nu_{3}, \nu_{4}\right) \\
+4 F_{21}\left(\nu_{1}, \nu_{2}\right) F_{21}\left(\nu_{3}, \nu_{4}\right)+12 F_{21}\left(\nu_{3}, \nu_{4}\right)^{2}+4 F_{21}\left(\nu_{1}, \nu_{2}\right) F_{21}\left(\nu_{5}, \nu_{6}\right)+4 F_{21}\left(\nu_{3}, \nu_{4}\right) F_{21}\left(\nu_{5}, \nu_{6}\right) \\
+12 F_{21}\left(\nu_{5}, \nu_{6}\right)^{2}+10 F_{01}\left(\nu_{3}, \nu_{4}\right)\left\{F_{01}\left(\nu_{5}, \nu_{6}\right)+2 F_{21}\left(\nu_{1}, \nu_{2}\right)+2 F_{21}\left(\nu_{5}, \nu_{6}\right)\right\} \\
\left.+10 F_{01}\left(\nu_{1}, \nu_{2}\right)\left\{F_{01}\left(\nu_{3}, \nu_{4}\right)+F_{01}\left(\nu_{5}, \nu_{6}\right)+2 F_{21}\left(\nu_{3}, \nu_{4}\right)+2 F_{21}\left(\nu_{5}, \nu_{6}\right)\right\}\right]
\end{array}
$$

After performing the radial integrations we should integrate over frequencies. The $\delta$-function in Eq. (130) annihilates one of the integrations. It is convenient to perform the other integrations after the transformation of the variables: $\omega_{1}=\frac{1}{4} \Delta E(1-x)(1+y)$, $\omega_{2}=\frac{1}{4} \Delta E(1-x)(1-y)$ and $\omega_{3}=\frac{1}{2} \Delta E(1+x)$. Here $\Delta E=E_{2 \mathrm{p}}-E_{1 \mathrm{~s}}=3 / 8$ in atomic units. The final result will be written in atomic units:

$$
\begin{array}{r}
W_{2 \mathrm{p} 1 \mathrm{~s}}^{(3)}=\int_{0}^{3 / 8} d \omega_{3} \int_{0}^{3 / 8-\omega_{3}} d \omega_{2} \int_{0}^{3 / 8-\omega_{3}-\omega_{2}} d \omega_{1} d W_{2 \mathrm{p} 1 \mathrm{~s}}^{(3)}=\frac{1}{3 !} \int_{0}^{3 / 8} \int_{0}^{3 / 8} \int_{0}^{3 / 8} d W_{2 \mathrm{p} 1 \mathrm{~s}}^{(3)}= \\
=\frac{1}{3 !}\left(\frac{3}{32}\right)^{2} \int_{-1}^{1} \int_{-1}^{1} d W_{2 \mathrm{p} 1 \mathrm{~s}}^{(3)} \frac{d x d y}{(1+y)(1-x)}=0.263466(1) \cdot 10^{-4} \alpha(\alpha Z)^{8} \text { a.u. }=6.391(1) \cdot 10^{-8} \mathrm{~s}^{-1} .
\end{array}
$$

For $Z=1$ the result is $W_{2 \mathrm{p} 1 \mathrm{~s}}^{(3)}=6.391(1) \cdot 10^{-8} \mathrm{~s}^{-1}$. The smallness of this result even compared to the two-photon $2 \mathrm{p}-1 \mathrm{~s}$ decays means that the 3-photon transitions cannot play any significant role for the astrophysical purposes, i.e. for the electron recombination history in the early Universe. For comparison we also provide the one-, two- and three-photon transition rates for transitions from $2 \mathrm{p}, 2 \mathrm{~s}$ levels (in r.u. and $\mathrm{s}^{-1}$, where $m$ is the electron mass): 
TABLE I: Electric (E1) and magnetic (M1) multi-photon transition rates between 2s, 2p and 1s levels are presented for hydrogen-like ions in relativistic units (r.u.) and for hydrogen in units $\mathrm{s}^{-1}$, respectively. $m$ and $Z$ denote the electron mass and nuclear charge number.

\begin{tabular}{lccr}
\hline transition & (type) & transition rates & \\
\hline $2 \mathrm{p} \rightarrow 1 \mathrm{~s}$ & (E1) & $3.902 \cdot 10^{-2} m \alpha(\alpha Z)^{4}$ r.u. & $6.25 \cdot 10^{8} \mathrm{~s}^{-1}$ \\
$2 \mathrm{~s} \rightarrow 1 \mathrm{~s}$ & $(\mathrm{E} 1 \mathrm{E} 1)$ & $1.317 \cdot 10^{-3} m \alpha^{2}(\alpha Z)^{6}$ r.u. & $8.229 \mathrm{~s}^{-1}$ \\
$2 \mathrm{p} \rightarrow 1 \mathrm{~s}$ & $(\mathrm{E} 1 \mathrm{E} 1 \mathrm{E} 1)$ & $2.635 \cdot 10^{-5} m \alpha^{3}(\alpha Z)^{8}$ r.u. & $6.39 \cdot 10^{-8} \mathrm{~s}^{-1}$ \\
$2 \mathrm{~s} \rightarrow 1 \mathrm{~s}$ & $(\mathrm{M} 1)$ & $\frac{1}{972} m \alpha(\alpha Z)^{10}$ r.u. & $2.5 \cdot 10^{-6} \mathrm{~s}^{-1}$ \\
$2 \mathrm{p} \rightarrow 1 \mathrm{~s}$ & $(\mathrm{E} 1 \mathrm{M} 1)$ & $2.911 \cdot 10^{-5} m \alpha^{2}(\alpha Z)^{8}$ r.u. & $9.68 \cdot 10^{-6} \mathrm{~s}^{-1}$ \\
$2 \mathrm{p} \rightarrow 1 \mathrm{~s}$ & $(\mathrm{E} 1 \mathrm{E} 2)$ & $1.989 \cdot 10^{-5} m \alpha^{2}(\alpha Z)^{8}$ r.u. & $6.612 \cdot 10^{-6} \mathrm{~s}^{-1}$ \\
\hline
\end{tabular}

\section{CONLUSIONS}

In this paper analytical results for $2 \mathrm{~s}, 2 \mathrm{p}$ levels decays for the hydrogen-like atomic systems with one-, two- and threephoton emission have been presented. All calculations were performed within Pauli approximation utilizing the Coulomb Green function. The emission processes were also calculated in the presence of an external electric field. For the two-photon decays in the absence of an external electric field the obtained results are in good agreement with those of other calculations.

The two-photon decays E1E2 and E1M1 were evaluated with different sets of quantum numbers (representations) for the emitted photon, namely, parity and momentum or polarization vector e and wave vector k. Moreover, we have employed different forms in combination with different gauges. The results do not differ in magnitude by more then $0.1 \%$ from fully relativistic values which were obtained earlier. Recently a paper [44] did arrive where, in particular, 2p-1s E1M1 and E1E2 transition rates were evaluated for the $\mathrm{H}$-like ions in the wide range of $Z$ values. For $Z=1$ the results of [44] are in agreement with our ones.

We have evaluated also the two-photon decay probabilities E1E2 and E1M1 with the set of quantum numbers e, $\mathbf{k}$ to investigate the probability dependence on directions of the photons emission. It allowed us to obtain the two-photon emission processes in the presence of the external electric field. In particular, we have demonstrated that interference terms in the E1E1 and E1E2, E1M1 transitions appear, which depend linearly on the external electric field.

The important result of our calculations is the prediction of a characteristic difference in transition probabilities (spectra) between $\mathrm{H}$ and $\overline{\mathrm{H}}$ atoms in the presence an external electric field, caused by the terms, linear in the electric field. This effect was not yet discussed in literature. The observation of this effect would also allow for drawbacks on our understanding of fundamental symmetries in nature, i.e. the CPT-symmetry: Any deviation from this result would provide a hint for CPT violation from a low-energy physics scenario, i.e. atoms in an external electric field.

Finally, we have compared the radiative correction evaluated in [12] with the electric field correction and determined the magnitude of the electric field strength, when both corrections become of the same order.

\section{Acknowledgements}

The work of D. S. and V. S. was supported by the Non-profit Foundation Dynasty (Moscow). D. S., L. L. and V. S. also acknowledge the support by RFBR grant grant Nr. 08-02-00026. V. S. acknoledges the support of St. Petersburg government. The authors acknowledge finacial support provided by DFG, BMBF and GSI. L. L. and D. S. acknowledge also the support by the Program of development of scientific potential of High School, Ministry of Education and Science of Russian Federation, grant $\aleph 2.1 .1 / 1136$.

[1] G. Gabrielse, V. N. S. Bowden, P. Oxley, A. Speck, C. H. Storry, J. N. Tan, M. Wessels, D. Grzonka, W. Oelert, G. Shepers, T. Sefzig, J. Walz, H. Pittner, T. W. Hänsch and E. A. Hessels, Phys. Rev. Lett. 89, 213401 (2002); 89, 23340 (2002).

[2] M. Amorreti et al, Nature (London) 419, 456 (2002).

[3] R. Bluhm, V. A. Kostelecký and N. Russell, Phys. Rev. Lett., 82(11), 2254 (1999).

[4] A. Huber, B. Gross, M. Weitz and T. W. Hänsch Phys. Rev. A 59, 1844 (1999).

[5] M. Niering, R. Holzwarth, J. Reichert, P. Pokasov, Th. Udem, M. Weitz, T. W. Hänsch, P. Lemond, G. Semtarelli, M. Abgrall, P. Lourent, C. Salomon and A. Clairon Phys. Rev. Lett. 84, 5496 (2000).

[6] L. Labzowsky and D. Solovyev, Phys. Rev. A 68, 014501 (2003).

[7] L. Labzowsky, D. Solovyev, V. Sharipov, G. Plunien and G. Soff, J. Phys. B 36, L227-L233 (2003).

[8] L. Labzowsky, V. Sharipov, D. Solovyev, G. Plunien and G. Soff, Int. J. Mod. Phys. B 18, 3875-3886 (2004).

[9] M. Göppert-Mayer, Ann. Phys. (Leipzig), 9, 273 (1931). 
[10] G. Breit and E. Teller, Astrophys. J. 91, 215 (1940).

[11] S. Klarsfeld, Phys. Lett. A 30, 382 (1969).

[12] U. D. Jenschura, Phys. Rev. A 69, 052118, (2004).

[13] R. W. Schmieder, Phys. Rev. A 7, 1458 (1973).

[14] L. N. Labzowsky and A. V. Shonin, Phys. Rev. A 69, 012503 (2004).

[15] L. N. Labzowsky, A. V. Shonin and D. A. Solovyev, J. Phys. B 38, 265 (2005).

[16] L. Hostler, J. Math. Phys. 5, 591 (1964).

[17] B. A. Zon and L. P. Rapoport, Pis'ma Zh. Eksp. Teor. Fiz. 7, 70 (1968); [Engl. Transl. JETP Lett. 7, 52 (1968)].

[18] L. P. Rapoport, B. A. Zon and N. L. Manakov, Teorija mnogofotonnych prozessov $v$ atomach, (Theory of the multiphoton processes in atoms) (Moscow, Atomizdat, 1978) (in Russian).

[19] E. J. Kelsey, J. Macek, J. Math. Phys. 17, 1182 (1976).

[20] J. P. Gasean, J. Math. Phys. 23, 156 (1982).

[21] G. W. F. Drake, Nucl. Instr. Meth. B 66, 465 (1985).

[22] S. G. Karshenboim, Zh. Eksp. Teor. Fiz. 107, 1061, (1995); [Engl. Transl: Sov. Phys. JETP 80, 593 (1995)].

[23] V. G. Ivanov and S. G. Karshenboim, Zh. Eksp. Teor. Fiz. 109, 1219 (1996); [Engl. Transl: Sov. Phys. JETP 80, 656 (1996)].

[24] L. Labzowsky, D. Solovyev, G. Plunien and G. Soff, Eur. Phys. J. D 37, 335 (2006).

[25] A. A. Nikitin, Z. B. Rudzikas, Osnovy teorii specktrov atomov i ionov (Foundations of the theory of spectra for atoms and ions), Moskva, Nauka, 1983 (in Russian).

[26] Ya. I. Azimov, A. A. Ansel'm, A. N. Moskalev and R. M. Ryndin, Zh. Exsp. Teor. Fiz. 67, 17 (1974); [Engl. Transl. Sov. Phys. - JETP, 40, 8 (1975)].

[27] P. J. Mohr, Phys. Rev. Lett. 40, 854 (1978).

[28] M. Hillery and P. J. Mohr, Phys. Rev. A 21, 24 (1980).

[29] J. Chluba and R. A. Sunyaev, Astronomy and Astrophysics, 446, 39 (2006).

[30] J. Chluba and R. A. Sunyaev, astro-ph/0608120

[31] L. Labzowsky, G. Shedrin, D. Solovyev and G. Plunien, Phys. Rev. Lett. 98, 203003 (2007).

[32] L. Labzowsky, G. Shedrin, D. Solovyev and G. Plunien, Can. J. Phys. 85, 585 (2007).

[33] D. A. Varshalovich, A. N. Moskalev and V. K. Khersonskii, Quantum Theory of Angular Momentum, World Scientific (1988) (English edition of the original Russian publication Nauka, Leningrad, 1975).

[34] G. W. F. Drake, Phys. Rev. A 3, 908 (1971).

[35] J. Sucher, Rep. Prog. Phys. 41, 1978.

[36] W. R. Johnson, Phys. Rev. Lett. 29, 1123 (1972).

[37] A. I. Akhiezer and V. B. Berestetskii, Quantum Electrodynamics, Wiley, New York (1965).

[38] H. A. Bethe and E. E. Salpeter, Quantum mehanics of one- and two-electron atoms, Springer (1957).

[39] Ya. B. Zeldovich, Zh. Exsp. Teor. Fiz. 34, 1483 (1960); [Engl. Transl.: Sov. Phys. JETP 12, 1030 (1961)].

[40] N. S. Kychkin, U. M. Kaniauskas, Z. B. Rudzikas - Liet. fiz. rinkinys, 14, 727 (1974) (in Russian).

[41] U. M. Kaniauskas, G. V.Merkelis, Z. B. Rudzikas, Liet. fiz. rinkinys, 19, 795 (1979) (in Russian).

[42] L. N. Labzowsky and D. Solovyev, In: Precision Physics of Simple Atomic Systems, Eds S. G. Karshenboim and V. B. Smirnov, (Springer, p. 15 (2003)).

[43] B. A. Zon, N. L. Manakov and L. P. Rapoport, Zh. Eksp. Teor. Fiz. 55, 924 (1968).

[44] P. Amaro, J. P. Santos, F. Parente, A. Surzhykov and P. Indelicato, arXiv:0904.0708v1 [physics.atom-ph] (2009) 